\title{
Glueball spectrum based on a rigorous three-dimensional relativistic equation for two-gluon bound states I: Derivation of the relativistic equation
}

\author{
Jun-Chen $\mathrm{Su}^{1 *, 2}$ and Jian-Xing Chen ${ }^{3}$ \\ 1. Department of Physics, Harbin Institute of technology, Harbin 150006, \\ People's Republic of China \\ 2. Center for Theoretical Physics, College of Physics, Jilin University \\ Changchun 130023, People's Republic of China \\ 3. Department of Physics, Peking University, Beijing 100871, People's \\ Republic of China
}

()

\begin{abstract}
A rigorous three-dimensional relativistic equation satisfied by two-gluon bound states is derived from the QCD with massive gluons. With the gluon fields and the quark fields being expanded in terms of the gluon multipole fields and the spherical Dirac spinors respectively, the equation is well established in the angular momentum representation and hence is much convenient for solving the problem of two-gluon glueball spectra. In particular, the interaction kernel in the equation is exactly derived and given a closed expression which includes all the interactions taking place in the two-gluon glueballs. The kernel contains only a few types of Green's functions and commutators. Therefore, it is not only easily calculated by the perturbation method, but also provides a suitable basis for nonperturbative investigations.

PACS numbers: 11.10.Qr, 11.10.St, 12.38.Aw, 14.80.-j

* Corresponding author. E-mail address: junchens@public.cc.jl.cn
\end{abstract}

\section{INTRODUCTION}

It is a prominent feature of Quantum Chromodynamics (QCD) that besides the quark-gluon interaction, there are also the interactions between gluons. The self-interaction of gluons suggests that the gluons may form glueballs (the bound states of gluons) through their interactions [1,2]. This is an essential prediction which is of decisive significance for testing QCD and, therefore, has been arising great interest in searching for the glueballs in experiment [3-7]. But, there have been no faithful evidence to be established so far for their existence [8-15]. On the other hand, the property, the mass spectrum, the production and decay of the glueballs have extensively been investigated theoretically. Many approaches were proposed to serve such investigations such as the potential model [16-18], the bag model [19-20], the sum rule [21], the Bethe-Salpeter (B-S) equation [22-24] and the lattice simulation [25-30]. However, the theoretical results given by different approaches are different and even contradictory with each other [31-32]. This situation is attributed mainly to the fact that the quark-gluon confinement has not clearly been understood so far. Commonly, it is believed that the lattice gauge approach would give a more reliable prediction because the approach is grounded on the first principle of QCD and essentially nonperterbative although certain approximations are inevitably made in practical calculations. In addition, it is widely recognized that the B-S equation which is set up on the basis of quantum field theory is a rigorous formalism for the bound state problem [33] and suitable to study the glueballs [23-24]. Nevertheless, there are two difficulties in the previous application of this equation. One difficulty arises from the interaction kernel in the equation. This kernel was not given a closed expression in the past although the expression can be derived by the procedure as demonstrated in a recent publication of one author of this paper [34]. Ordinarily, the kernel is defined by a sum of all two-particle irreducible Feynman graphs and can only be calculated by the perturbation method. Another difficulty is ascribed to the four-dimensional nature of the equation in which the relative time (or the relative energy) is unphysical and would lead to unphysical solutions [35]. So, many efforts in the past are paid to recast the equation into a three-dimensional one in the instantaneous approximation[36] or the quasipotential approach [37-38].

In this paper, we are devoted to deriving a rigorous three-dimensional relativistic equation satisfied by two-gluon bound states so as to provide a firm basis for further study. A practical application of this equation to calculate the glueball spectrum will be presented in the next paper. The distinctive features of the equation derived are as follows. (1) The equation is exactly relativistic, containing all the retardation effect in it, unlike the B-S equation given in the instantaneous approximation in which the retardation effect is completely neglected. The equation is derived in the equal-time formalism by the consideration that a bound state is space-like and can exists in the equal-time Lorentz frame. In this frame, the relativistic equation naturally becomes a three-dimensional one without loss of any rigorism. Moreover, different from the B-S equation, the three-dimensional equation derived in this paper is a standard eigenequation of Schrödinger-type. In the position space, it appears to be first-order differential equations. In particular, 
the interaction kernel in the equation is given a closed expression which is derived by the procedure proposed first in Ref.[39] for a two-fermion system and subsequently demonstrated in Ref.[40] for quark-antiquark bound states. The kernel derived contains exactly all the interactions taking place in the bound states and is represented in terms of only a few types of Green's functions. Such a kernel can not only be easily calculated by the perturbation method, but also is suitable for nonperturbative investigations. (2) The three-dimensional equation is established in the angular momentum representation. Since a glueball is the state of definite spin and parity, obviously, to investigate the glueballs, it is much more convenient to work in the angular momentum representation. In order to express the relativistic equation in such a representation, it is necessary to express the QCD in the same representation. This can be done by expanding the quantized gluon and quark fields in terms of the gluon multipole fields and the spherical Dirac spinors, respectively [41-42]. With these expansions, the vertices in the interaction Hamiltonian can all be given explicit and analytical expressions, as will be shown in the next paper. This achievement is due to that the integrals containing three and four spherical Bessel functions in the vertices are all calculated analytically and expressed explicitly. We would like to note that in comparison with the momentum representation in which every glueball state must be separately constructed according to a certain requirement for the Lorentz and CTP transformation properties [24] and the vertices would involve the gluon polarization vectors which are not convenient to deal with, the angular momentum representation has an advantage that the glueball state can easily be written out in a consistent manner and the QCD vertices exhibit the spin structures much clearly.(3) The relativistic equation is set up by starting from the QCD with massive gluons. According to the conventional concept of QCD, in order to keep the Lagrangian to be gauge-invariant, the gluons must be massless. On the contrary, in most of the previous investigations of the glueballs, an effective gluon mass was phenomenologically introduced so as to get reasonable theoretical results $[17,18,23,24]$. The gluon mass was supposed to be generated dynamically from the interaction with the physical vacuum of the Yang-Mills theory [23] or through strong gluon-binding force [17]. Apparently, these arguments would not be considered to be stringent and logically consistent with the concept of the ordinary QCD. One of the authors of this paper in his recent article [43] gave a different reasoning that the QCD with massive gluons can, actually, be set up on the principle of gauge-invariance without the need of introducing the Higgs mechanism or the Stückelberg fields. The essential points to achieve this conclusion are: (a) The gluon fields must be viewed as a constrained system in the whole space of vector potentials and the Lorentz condition, as a necessary constraint, must be introduced from the beginning and imposed on the Lagrangian; (b) The gauge-invariance of a gauge field should be generally examined from the action of the field other than from the Lagrangian because the action is of more fundamental dynamical meaning than the Lagrangian. Particularly, for a constrained system such as the gluon field, the gauge-invariance should be seen from its action given in the physical space defined by the Lorentz condition. This concept is well-known in Mechanics; (c) In the physical space, only infinitesimal gauge transformations are possibly allowed and necessary to be considered. This fact was clarified originally in Ref.[44]. Based on these points of view, it is easy to prove that the QCD with massive gluons is gauge-invariant. Moreover, the renormalizability and unitarity of the theory have been proved to be no problems [45].

The remainder of this paper is arranged as follows. In Section II, the massive QCD and its Lagrangian are briefly described. Section III is used to formulate the angular momentum representation and give the expansions for vector, spinor and ghost fields in this representation. In Section IV, the expression of QCD Hamiltonian in the angular momentum space will be described and discussed. Section V serves to derive the three-dimensional relativistic equation satisfied by the glueballs. In Section VI, we are devoted to derive a closed expression of the interaction kernel included in the relativistic equation. In the last section, some remarks will be made. In Appendix, we present a breif derivation of the spherical Dirac spinors given in the angular momentum representation.

\section{QCD LAGRANGIAN WITH MASSIVE GLUONS}

In the previous attempt of building up the massive non-Abelian gauge field theory, the following massive Yang-Mills Lagrangian density was chosen to be the starting point $[43,46]$ :

$$
\mathcal{L}=-\frac{1}{4} F^{a \mu \nu} F_{\mu \nu}^{a}+\frac{1}{2} \mu^{2} A^{a \mu} A_{\mu}^{a},
$$

where $A_{\mu}^{a}$ is the vector potential for a gluon field,

$$
F_{\mu \nu}^{a}=\partial_{\mu} A_{\nu}^{a}-\partial_{\nu} A_{\mu}^{a}+g f^{a b c} A_{\mu}^{b} A_{\nu}^{c}
$$

is the field strength tensor in which $g$ is the QCD coupling constant and $f^{a b c}$ are the structure constants of color $\mathrm{SU}(3)$ group and $\mu$ is the gluon mass. The first term in the Lagrangian is the ordinary Yang-Mills Lagrangian which 
is gauge-invariant under the whole Lie group and used to determine the form of interactions among the gluon fields themselves. The second term in the Lagrangian is the mass term which is not gauge-invariant and only affects the kinematic property of the fields. The above Lagrangian itself was ever considered to give a complete description of the massive gauge field dynamics. This consideration is not correct because the Lagrangian is not only not gaugeinvariant, but also contains redundant unphysical degrees of freedom which must be eliminated by introducing a suitable constraint condition. As we know, a massive gauge field has three polarization states which need only three spatial components of the four-dimensional vector potential $A_{\mu}^{a}$ to describe them. In Lorentz-covariant formulation, a full vector potential $A^{\mu}$ can be split into two Lorentz-covariant parts: the transverse vector potential $A_{T}^{\mu}$ and the longitudinal vector potential $A_{L}^{\mu}$

$$
A^{\mu}=A_{T}^{\mu}+A_{L}^{\mu}
$$

Since the Lorentz-covariant transverse vector potential $A_{T}^{a \mu}$ contains three-independent spatial components, it is sufficient to represent the polarization states of a massive gluon. Whereas, the Lorentz-covariant longitudinal vector potential $A_{L}^{a \mu}$ appears to be a redundant unphysical variable which must be constrained by introducing the Lorentz condition

$$
\partial^{\mu} A_{\mu}^{a}=0,
$$

whose solution is

$$
A_{L}^{a \mu}=0
$$

With this solution, the massive Yang-Mills Lagrangian may be expressed in terms of the independent dynamical variables $A_{T}^{a \mu}$

$$
\mathcal{L}=-\frac{1}{4} F_{T}^{a \mu \nu} F_{T \mu \nu}^{a}+\frac{1}{2} \mu^{2} A_{T}^{a \mu} A_{T \mu}^{a}
$$

which gives a complete description of the massive gluon field dynamics. If we want to represent the dynamics in the whole space of the full vector potential as described by the massive Yang-Mills Lagrangian in Eq.(2.1), the massive gluon field must be treated as a constrained system. In this case, the Lorentz condition in Eq.(2.4), as a constraint, is necessarily introduced from the onset and imposed on the Lagrangian in Eq.(2.1) so as to guarantee the redundant degrees of freedom to be eliminated from the Lagrangian. Since the action is of more dynamical significance than the Lagrangian, the gauge-invariance of QCD should generally be seen from the action given by the Lagrangian in Eq.(2.6) or the Lagrangian in Eq.(2.1) constrained by the Lorentz condition in Eq.(2.4). Under the gauge transformations

$$
\delta A_{\mu}^{a}=D_{\mu}^{a b} \theta^{b}
$$

where

$$
D_{\mu}^{a b}=\delta^{a b} \partial_{\mu}-g f^{a b c} A_{\mu}^{c} .
$$

Noticing the identity $f^{a b c} A^{a \mu} A_{\mu}^{b}=0$, it is easy to prove that the action given by the Lagrangian in Eq.(2.1) and constrained by he Lorentz condition in Eq.(2.4) is gauge-invariant,

$$
\delta S=\int d^{4} x \delta \mathcal{L}=-\mu^{2} \int d^{4} x \theta^{a} \partial^{\mu} A_{\mu}^{a}=0 .
$$

This suggests that the QCD with massive gluons may also be set up on the basis of gauge-invariance principle.

Now, let us briefly describe quantization of the QCD with massive gluons, This quantization was carried out by different approaches in Ref.[43]. A simpler quantization is performed in the Lagrangian path-integral formalism by means of the Lagrange undetermined multiplier method which was shown to be equivalent to the Faddeev-Popov approach of quantization [44]. For this quantization, it is convenient to generalize the QCD Lagrangian and the Lorentz condition to the following forms:

$$
\begin{aligned}
\mathcal{L}_{\lambda}= & \bar{\psi}\left\{i \gamma^{\mu}\left(\partial_{\mu}-i g T^{a} A_{\mu}^{a}\right)-m\right\} \psi-\frac{1}{4} F^{a \mu \nu} F_{\mu \nu}^{a} \\
& +\frac{1}{2} \mu^{2} A^{a \mu} A_{\mu}^{a}-\frac{1}{2} \alpha\left(\lambda^{a}\right)^{2}
\end{aligned}
$$


and

$$
\partial^{\mu} A_{\mu}^{a}+\alpha \lambda^{a}=0
$$

where for completeness, the quark fields have been included in the Lagrangian in which $\bar{\psi}$ and $\psi$ stand for the quark fields, $T^{a}$ are the color matrices and $m$ is the quark mass, $\lambda^{a}(x)$ are the extra functions which will be identified with the Lagrange multipliers and $\alpha$ is an arbitrary constant playing the role of gauge parameter. According to the general procedure for constrained systems, the constraint in Eq.(2.11) may be incorporated into the Lagrangian in Eq.(2.10) by the Lagrange multiplier method, giving a generalized Lagrangian such that

$$
\begin{aligned}
\mathcal{L}_{\lambda}= & \bar{\psi}\left\{i \gamma^{\mu}\left(\partial_{\mu}-i g T^{a} A_{\mu}^{a}\right)-m\right\} \psi-\frac{1}{4} F^{a \mu \nu} F_{\mu \nu}^{a}+\frac{1}{2} \mu^{2} A^{a \mu} A_{\mu}^{a} \\
& -\frac{1}{2} \alpha\left(\lambda^{a}\right)^{2}+\lambda^{a}\left(\partial^{\mu} A_{\mu}^{a}+\alpha \lambda^{a}\right) \\
= & \bar{\psi}\left\{i \gamma^{\mu}\left(\partial_{\mu}-i g T^{a} A_{\mu}^{a}\right)-m\right\} \psi-\frac{1}{4} F^{a \mu \nu} F_{\mu \nu}^{a}+\frac{1}{2} \mu^{2} A^{a \mu} A_{\mu}^{a} \\
& +\lambda^{a} \partial^{\mu} A_{\mu}^{a}+\frac{1}{2} \alpha\left(\lambda^{a}\right)^{2} .
\end{aligned}
$$

This Lagrangian is obviously not gauge-invariant. However, for building up a correct gauge field theory, it is necessary to require the dynamics of the system, i.e. the action given by the Lagrangian (2.12) to be invariant under the gauge transformations denoted in Eqs.(2.7) and (2.8). By this requirement, noticing the identity $f^{a b c} A^{a \mu} A_{\mu}^{b}=0$ and applying the constraint condition in Eq.(2.11), we find

$$
\delta S_{\lambda}=-\frac{1}{\alpha} \int d^{4} x \partial^{\nu} A_{\nu}^{a}(x) \partial^{\mu}\left(\mathcal{D}_{\mu}^{a b}(x) \theta^{b}(x)\right)=0,
$$

where

$$
\mathcal{D}_{\mu}^{a b}(x)=\delta^{a b} \frac{\sigma^{2}}{\square_{x}} \partial_{\mu}^{x}+D_{\mu}^{a b}(x),
$$

in which $\sigma^{2}=\alpha \mu^{2}$ and $D_{\mu}^{a b}(x)$ was defined in Eq.(2.8). From equation (2.11) we see $\frac{1}{\alpha} \partial^{\nu} A_{\nu}^{a}=-\lambda^{a} \neq 0$. Therefore, to ensure the action to be gauge-invariant, the following constraint condition on the gauge group is necessary to be required

$$
\partial_{x}^{\mu}\left(\mathcal{D}_{\mu}^{a b}(x) \theta^{b}(x)\right)=0 .
$$

These are the coupled equations satisfied by the parametric functions $\theta^{a}(x)$ of the gauge group. Since the Jacobian is not singular

$$
\operatorname{det} M \neq 0,
$$

where

$$
\begin{aligned}
M^{a b}(x, y) & =\left.\frac{\delta\left(\partial_{x}^{\mu} \mathcal{D}_{\mu}^{a c}(x) \theta^{c}(x)\right)}{\delta \theta^{b}(y)}\right|_{\theta=0} \\
& =\delta^{a b}\left(\square_{x}+\sigma^{2}\right) \delta^{4}(x-y)-g f^{a b c} \partial_{x}^{\mu}\left(A_{\mu}^{c}(x) \delta^{4}(x-y)\right),
\end{aligned}
$$

the above equations are solvable and would give a set of solutions which express the parametric functions $\theta^{a}(x)$ as functionals of the vector potentials $A_{\mu}^{a}(x)$. The constraint conditions in equation (2.15) may also be incorporated into the Lagrangian (2.12) by the Lagrange undetermined multiplier method. In doing this, it is convenient, as usually done, to introduce ghost field variables $C^{a}(x)$ in such a fashion

$$
\theta^{a}(x)=\xi C^{a}(x),
$$

where $\xi$ is an infinitesimal Grassmann's number. In accordance with Eq.(2.18), the constraint condition in Eq.(2.15) can be rewritten as

$$
\partial^{\mu}\left(\mathcal{D}_{\mu}^{a b} C^{b}\right)=0,
$$


where the number $\xi$ has been dropped. This constraint condition usually is called ghost equation. When the condition in Eq.(2.19) is incorporated into the Lagrangian in Eq.(2.12) by the Lagrange multiplier method, we obtain a more generalized Lagrangian as follows

$$
\begin{aligned}
\mathcal{L}_{\lambda}= & \bar{\psi}\left\{i \gamma^{\mu}\left(\partial_{\mu}-i g T^{a} A_{\mu}^{a}\right)-m\right\} \psi-\frac{1}{4} F^{a \mu \nu} F_{\mu \nu}^{a}+\frac{1}{2} \mu^{2} A^{a \mu} A_{\mu}^{a} \\
& +\lambda^{a} \partial^{\mu} A_{\mu}^{a}+\frac{1}{2} \alpha\left(\lambda^{a}\right)^{2}+\bar{C}^{a} \partial^{\mu}\left(\mathcal{D}_{\mu}^{a b} C^{b}\right)
\end{aligned}
$$

where $\bar{C}^{a}(x)$, acting as Lagrange undetermined multipliers, are the new scalar variables conjugate to the ghost variables $C^{a}(x)$.

At present, we are ready to formulate the quantization of the QCD with massive gluons. As we learn from the Lagrange undetermined multiplier method, the dynamical and constrained variables as well as the Lagrange multipliers in the Lagrangian (2.20) can all be treated as free ones, varying arbitrarily. Therefore, we are allowed to use this kind of Lagrangian to construct the generating functional of Green's functions

$$
\begin{aligned}
& Z\left[J^{a \mu}, \bar{\eta}, \eta, \bar{\xi}^{a}, \xi^{a}\right] \\
= & \frac{1}{N} \int D\left(A_{\mu}^{a}, \bar{\psi}, \psi, \bar{C}^{a}, C^{a}, \lambda^{a}\right) \exp \left\{i \int d ^ { 4 } x \left[\mathcal{L}_{\lambda}(x)+J^{a \mu}(x) A_{\mu}^{a}(x)\right.\right. \\
& \left.\left.+\bar{\psi} \eta+\bar{\eta} \psi+\bar{\xi}^{a}(x) C^{a}(x)+\bar{C}^{a}(x) \xi^{a}(x)\right]\right\},
\end{aligned}
$$

where $D\left(A_{\mu}^{a}, \cdots, \lambda^{a}\right)$ denotes the functional integration measure, $J_{\mu}^{a}, \bar{\eta}, \eta, \bar{\xi}^{a}$ and $\xi^{a}$ are the external sources coupled to the gluon, quark and ghost fields and $N$ is a normalization constant. Looking at the expression of the Lagrangian (2.20), we see, the integral over $\lambda^{a}(x)$ is of Gaussian-type. Upon completing the calculation of this integral, we finally arrive at [43]

$$
\begin{aligned}
& Z\left[J^{a \mu}, \bar{\eta}, \eta, \bar{\xi}^{a}, \xi^{a}\right] \\
= & \frac{1}{N} \int D\left(A_{\mu}^{a}, \bar{\psi}, \psi, \bar{C}^{a}, C^{a},\right) \exp \left\{i \int d ^ { 4 } x \left[\mathcal{L}_{e f f}(x)\right.\right. \\
& \left.\left.\quad+J^{a \mu}(x) A_{\mu}^{a}(x)+\bar{\psi} \eta+\bar{\eta} \psi+\bar{\xi}^{a}(x) C^{a}(x)+\bar{C}^{a}(x) \xi^{a}(x)\right]\right\},
\end{aligned}
$$

where

$$
\begin{aligned}
\mathcal{L}_{e f f}= & \bar{\psi}\left\{i \gamma^{\mu}\left(\partial_{\mu}-i g T^{a} A_{\mu}^{a}\right)-m\right\} \psi-\frac{1}{4} F^{a \mu \nu} F_{\mu \nu}^{a}+\frac{1}{2} \mu^{2} A^{a \mu} A_{\mu}^{a} \\
& -\frac{1}{2 \alpha}\left(\partial^{\mu} A_{\mu}^{a}\right)^{2}-\partial^{\mu} \bar{C}^{a} \mathcal{D}_{\mu}^{a b} C^{b}
\end{aligned}
$$

is the effective Lagrangian given in the general gauges.

From the generating functional shown in Eqs.(2.22) and ( 2.23), one may derive the free gluon propagator as follows

$$
i D_{\mu \nu}^{c d}(k)=-\frac{i \delta^{c d}}{k^{2}-\mu^{2}+i \varepsilon}\left[g_{\mu \nu}-(1-\alpha) \frac{k_{\mu} k_{\nu}}{k^{2}-\sigma^{2}+i \varepsilon}\right] .
$$

It is emphasized that when the gluon mass tends to zero, this propagator together with the effective Lagrangian in Eq.(2.23) and the generating functional in Eq.(2.22) all immediately go over to the results given in the QCD with massless gluons.

We would like to point out that the above propagator may also be derived from the Lagrangian in Eq.(2.23) by the method of canonical quantization[46]. In doing this, we need to use the Fourier representation of the free gluon field operator[46]

$$
\begin{aligned}
\mathbf{A}_{\mu}^{c}(x)= & \int \frac{d^{3} k}{(2 \pi)^{3 / 2}}\left\{\frac{1}{2 \omega(\vec{k})} \sum_{\lambda=1}^{3}\left[\epsilon_{\mu}^{\lambda}(\vec{k}) \mathbf{a}_{\lambda}^{c}(\vec{k}) e^{-i k x}+\epsilon_{\mu}^{\lambda *}(\vec{k}) \mathbf{a}_{\lambda}^{c+}(\vec{k}) e^{i k x}\right]\right. \\
& \frac{1}{2 \omega_{0}(\vec{k})} \frac{\widetilde{k}}{\mu}\left[\mathbf{a}_{0}^{c}(\vec{k}) e^{-i \widetilde{k} x}+\mathbf{a}_{0}^{c+}(\vec{k}) e^{i \widetilde{k} x}\right],
\end{aligned}
$$

where $k=\left(k_{0}, \vec{k}\right)$ and $\widetilde{k}=\left(\widetilde{k}_{0}, \vec{k}\right)$ with $k_{0} \equiv \omega(\vec{k})=\sqrt{\vec{k}^{2}+\mu^{2}}$ and $\widetilde{k}_{0} \equiv \omega_{0}(\vec{k})=\sqrt{\vec{k}^{2}+\sigma^{2}}, \epsilon_{\mu}^{\lambda}(\vec{k})$ are the polarization vectors satisfying the transversity condition 


$$
k^{\mu} \epsilon_{\mu}^{\lambda}(\vec{k})=0
$$

which means that only three spatial components of $\epsilon_{\mu}^{\lambda}(\vec{k})$ are independent and the creation and annihilation operators $\mathbf{a}_{\lambda}^{c}(\vec{k})$ and $\mathbf{a}_{\lambda}^{c+}(\vec{k})(\lambda=0,1,2,3)$ are subject to the following commutation relations

$$
\begin{aligned}
{\left[\mathbf{a}_{\lambda}^{c}(\vec{k}), \mathbf{a}_{\lambda^{\prime}}^{d+}\left(\overrightarrow{k^{\prime}}\right)\right] } & =-\delta^{c d} g^{\lambda \lambda^{\prime}} \delta^{3}\left(\vec{k}-\overrightarrow{k^{\prime}}\right), \\
{\left[\mathbf{a}_{\lambda}^{c}(\vec{k}), \mathbf{a}_{\lambda^{\prime}}^{d}\left(\overrightarrow{k^{\prime}}\right)\right] } & =\left[\mathbf{a}_{\lambda}^{c+}(\vec{k}), \mathbf{a}_{\lambda^{\prime}}^{d+}\left(\overrightarrow{k^{\prime}}\right)\right]=0 .
\end{aligned}
$$

By making use of the expression in Eq.(2.25) and the above commutation relations, it is not difficult to derive

$$
i D_{\mu \nu}^{c d}(x-y)=\left\langle 0\left|T\left\{\mathbf{A}_{\mu}^{c}(x) \mathbf{A}_{\nu}^{d}(y)\right\}\right| 0\right\rangle=\int \frac{d^{4} k}{(2 \pi)^{4}} i D_{\mu \nu}^{c d}(k) e^{-i k(x-y)},
$$

where $i D_{\mu \nu}^{c d}(k)$ is just as that written in Eq.(2.24).

The propagator in Eq.(2.24) is written in arbitrary gauges. It has been proved that the S-matrices given by the QCD with massive gluons is independent of the gauge parameter $\alpha$ [45]. For example, in the tree diagram approximation, noticing the transversity condition denoted in Eq.(2.26) and the on-shell property of the gluon states, it is easy to verify that the $\alpha$-dependent term proportional to $k_{\mu} k_{\nu}$ in Eq.(2.24) gives no contribution to the S-matrix elements. This fact was early pointed out in Ref.[17]. In view of this fact, we may simply take the Feynman gauge $(\alpha=1)$ in practical calculations. In this gauge, the effective Lagrangian in Eq.(2.23) becomes

$$
\mathcal{L}=\mathcal{L}_{0}+\mathcal{L}_{I},
$$

where

$$
\mathcal{L}_{0}=\frac{1}{2} A_{\nu}^{a}\left(\square+\mu^{2}\right) A^{a \nu}+\bar{\psi}\left(i \gamma^{\mu} \partial_{\mu}-m\right) \psi+\bar{C}^{a}\left(\square+\mu^{2}\right) C^{a}
$$

and

$$
\begin{aligned}
\mathcal{L}_{I}= & -\frac{1}{2} g f^{a b c}\left(\partial_{\mu} A_{\nu}^{a}-\partial_{\nu} A_{\mu}^{a}\right) A^{b \mu} A^{c \nu}-\frac{1}{4} g^{2} f^{a b c} f^{a d e} A^{b \mu} A^{c \nu} A_{\mu}^{d} A_{\nu}^{e} \\
& +\bar{\psi} \gamma^{\mu} T^{a} \psi A_{\mu}^{a}+g f^{a b c} \partial^{\mu} \bar{C}^{a} C^{b} A_{\mu}^{c}
\end{aligned}
$$

are the free and interaction parts of the Lagrangian respectively. Correspondingly, the gluon propagator in Eq.(2.24) is reduced to

$$
i D_{\mu \nu}^{c d}(k)=-\frac{i \delta^{c d} g_{\mu \nu}}{k^{2}-\mu^{2}+i \varepsilon}
$$

\section{EXPRESSIONS OF THE QCD FIELDS IN ANGULAR MOMENTUM REPRESENTATION}

The fields in the Lagrangian in Eqs.(2.30) and (2.31) may be expressed in the angular momentum representation in terms of the eigenfunctions of total angular momenta for the vector, spinor and scalar fields. These eigenfunctions are described below. For the vector field, the complete set of the eigenfunctions were already found in the literature[41,42]. They include the scalar multipole field $A_{J M}^{S}(k \vec{x})$ and the vectorial multipole fields $\vec{A}_{J M}^{M}\left(k \vec{x}^{\prime}, \vec{A}_{J M}^{E}(k \vec{x})\right.$ and $\vec{A}_{J M}^{L}(k \vec{x})$. They are displayed in the following.

$$
\begin{aligned}
A_{J M}^{S}(k \vec{x}) & =\sqrt{\frac{2}{\pi}} k j_{J}(k r) Y_{J M}(\widehat{x}), \\
\vec{A}_{J M}^{M}(k \vec{x}) & =\frac{-i}{\sqrt{J(J+1)}} \widehat{L} A_{J M}^{S}(k \vec{x}) \\
& =\sqrt{\frac{2}{\pi}} k j_{J}(k r) \vec{Y}_{J J M}(\widehat{x}),
\end{aligned}
$$




$$
\begin{aligned}
\vec{A}_{J M}^{E}(k \vec{x})= & \frac{1}{k \sqrt{J(J+1)}} \nabla \times \widehat{L} A_{J M}^{S}(k \vec{x}) \\
= & -i \sqrt{\frac{2}{\pi} \frac{k}{\sqrt{2 J+1}}}\left[\sqrt{J} j_{J+1}(k r) \vec{Y}_{J J+1 M}(\widehat{x})\right. \\
& \left.-\sqrt{J+1} j_{J-1}(k r) \vec{Y}_{J J-1 M}(\widehat{x})\right], \\
\vec{A}_{J M}^{L}(k \vec{x})= & \frac{-i}{k} \nabla A_{J M}^{S}(k \vec{x}) \\
= & -i \sqrt{\frac{2}{\pi}} \frac{k}{\sqrt{2 J+1}}\left[\sqrt{J+1} j_{J+1}(k r) \vec{Y}_{J J+1 M}(\widehat{x})\right. \\
& \left.+\sqrt{J} j_{J-1}(k r) \vec{Y}_{J J-1 M}(\widehat{x})\right],
\end{aligned}
$$

where $J, M$ mark the total angular momentum and its third component of the vector field eigenfunction, $\vec{k}$ is the momentum of a single particle, $k=|\vec{k}|, j_{l}(k r)$ is the $l$-th spherical Bessel function with $r=|\vec{x}|, \widehat{L}=-i \vec{x} \times \nabla$ is the orbital angular momentum operator and $\vec{Y}_{J l M}(\widehat{x})$ denotes the vectorial spherical harmonic function with total angular momentum $J M$ and orbital angular momentum $l=J-1, J, J+1$. This function is defined as

$$
\vec{Y}_{J l M}(\widehat{x})=\sum_{m q} C_{l m 1 q}^{J M} Y_{l m}(\widehat{x}) \vec{e}_{q}
$$

where $C_{l m 1 q}^{J M}$ are the Clebsch-Gordan $(\mathrm{C}-\mathrm{G})$ coefficients, $Y_{l m}(\widehat{x})$ with $\widehat{x}=(\theta, \phi)$ are the eigenfunction of orbital angular momentum operator $\widehat{L}$ and $\vec{e}_{q}$ is the eigenfunction of the spin operator for a vector particle. Since the function defined by

$$
\vec{A}_{J M}(\vec{x})=j_{J}(k r) \vec{Y}_{J l M}(\widehat{x})
$$

and

$$
A_{J M}^{0}(\vec{x})=j_{J}(k r) Y_{J M}(\widehat{x})
$$

respectively satisfy the following equations of motion derived from the first term of the Lagrangian in Eq.(2.30)

$$
\left(\square+\mu^{2}\right) \vec{A}=0
$$

and

$$
\left(\square+\mu^{2}\right) A^{0}=0
$$

as we see, the functions in Eqs.(3.1)-(3.4) completely describe the eigenstates of the total angular momentum for a free massive gluon field (or for a massless gluon field when the mass $\mu=0$ ). Furthermore, as the functions $\vec{A}_{J M}^{M}(k \vec{x})$ and $\vec{A}_{J M}^{E}(k \vec{x})$ satisfy the transversity condition

$$
\nabla \cdot \vec{A}=0
$$

and are related to each other by

$$
\nabla \times \vec{A}_{J M}^{M}(k \vec{x})=k \vec{A}_{J M}^{E}(k \vec{x}),
$$

the $\vec{A}_{J M}^{M}(k \vec{x})$ is usually called transverse magnetic multipole field and $\vec{A}_{J M}^{E}(k \vec{x})$ transverse electric multipole field. While the field $\vec{A}_{J M}^{L}(k \vec{x})$ is referred to as longitudinal multipole field because it obeys the condition of longitudinal fields $\nabla \times \vec{A}=0$. It is easy to verify that these vectorial eigenfunctions meet the orthogonality relation

$$
\int d^{3} x \vec{A}_{J M}^{\lambda}(k \vec{x}) \vec{A}_{J^{\prime} M^{\prime}}^{\lambda^{\prime}}\left(k^{\prime} \vec{x}\right)=\delta_{\lambda \lambda^{\prime}} \delta\left(k-k^{\prime}\right) \delta_{J J^{\prime}} \delta_{M M^{\prime}},
$$


where $\lambda, \lambda^{\prime}=M, E, L$ label the different modes of the multipole fields. Similarly, for the scalar multipole field, we have

$$
\int d^{3} x A_{J M}^{s}(k \vec{x}) A_{J^{\prime} M^{\prime}}^{s}(k \vec{x})=\delta\left(k-k^{\prime}\right) \delta_{J J^{\prime}} \delta_{M M^{\prime}}
$$

Therefore, the multipole fields defined in Eqs.(3.1)-(3.4) may suitably be chosen as the basis functions to establish the angular momentum representation for the gluon fields. A gluon field operator $\mathbf{A}_{\mu}^{c}(x)$ may be expanded as

$$
\overrightarrow{\mathbf{A}}^{c}(x)=\sum_{\lambda J M} \int_{0}^{\infty} \frac{d k}{\sqrt{2 \omega}}\left[\mathbf{a}_{J M}^{c \lambda}(k) \vec{A}_{J M}^{\lambda}(k \vec{x}) e^{-i \omega t}+\mathbf{a}_{J M}^{c \lambda^{+}}(k) \vec{A}_{J M}^{\lambda^{*}}(k \vec{x}) e^{i \omega t}\right],
$$

where $\lambda=M, E, L$,

$$
\mathbf{A}_{0}^{c}(x)=\sum_{J M} \int_{0}^{\infty} \frac{d k}{\sqrt{2 \omega}}\left[\mathbf{a}_{J M}^{c s}(k) A_{J M}^{s}(k \vec{x}) e^{-i \omega t}+\mathbf{a}_{J M}^{c s+}(k) A_{J M}^{s^{*}}(k \vec{x}) e^{i \omega t}\right] .
$$

The creation and annihilation operators in the above expansions satisfy the following commutation relations

$$
\begin{aligned}
& {\left[\mathbf{a}_{J M}^{c \lambda}(k), \mathbf{a}_{J^{\prime} M^{\prime}}^{c^{\prime} \lambda^{\prime+}}\left(k^{\prime}\right)\right]=\delta\left(k-k^{\prime}\right) \delta_{c c^{\prime}} \delta_{\lambda \lambda^{\prime}} \delta_{J J^{\prime}} \delta_{M M^{\prime}},} \\
& {\left[\mathbf{a}_{J M}^{c \lambda}(k), \mathbf{a}_{J^{\prime} M^{\prime}}^{c^{\prime} \lambda^{\prime}}\left(k^{\prime}\right)\right]=\left[\mathbf{a}_{J M}^{c \lambda+}(k), \mathbf{a}_{J^{\prime} M^{\prime}}^{c^{\prime} \lambda^{\prime}+}\left(k^{\prime}\right)\right]=0,} \\
& {\left[\mathbf{a}_{J M}^{c s}(k), \mathbf{a}_{J^{\prime} M^{\prime}}^{c^{\prime} s^{\prime+}}\left(k^{\prime}\right)\right]=\delta\left(k-k^{\prime}\right) \delta_{c c^{\prime}} \delta_{J J^{\prime}} \delta_{M M^{\prime \prime}},} \\
& {\left[\mathbf{a}_{J M}^{c s}(k), \mathbf{a}_{J^{\prime} M^{\prime}}^{c^{\prime} s^{\prime}}\left(k^{\prime}\right)\right]=\left[\mathbf{a}_{J M}^{c s+}(k), \mathbf{a}_{J^{\prime} M^{\prime}}^{c^{\prime} s^{\prime+}}\left(k^{\prime}\right)\right]=0 .}
\end{aligned}
$$

From the transformation of a gluon field under the space inversion [46]

$$
P \overrightarrow{\mathbf{A}}^{c}(t, \vec{x}) P^{-1}=-\overrightarrow{\mathbf{A}}^{c}(t,-\vec{x}), P \mathbf{A}_{o}^{c}(t, \vec{x}) P^{-1}=\mathbf{A}_{o}^{c}(t,-\vec{x}),
$$

where $P$ is the space inversion operator and the parity of the multipole fields

$$
A_{J M}^{S}(-k \vec{x})=(-1)^{J} A_{J M}^{S}(k \vec{x}), \vec{A}_{J M}^{\lambda}(-k \vec{x})=(-1)^{J+\pi_{\lambda}} \vec{A}_{J M}^{\lambda}(k \vec{x}),
$$

where $\pi_{\lambda}=0$ if $\lambda=M$ and $\pi_{\lambda}=1$ if $\lambda=E, L$, one may find from Eqs.(3.14) and (3.15) the parities of the annihilation operators

$$
P \mathbf{a}_{J M}^{c s}(k) P^{-1}=(-1)^{J} \mathbf{a}_{J M}^{c s}(k), P \mathbf{a}_{J M}^{c \lambda}(k) P^{-1}=(-1)^{J+1+\pi_{\lambda}} \mathbf{a}_{J M}^{c \lambda}(k)
$$

and the same parity for the creation operators. In addition, from the charge conjugation of the gluon field $C A_{\mu}^{c}(t, \vec{x}) C^{-1}=-A_{\mu}^{c}(t, \vec{x})$, we obtain a minus C-parity for the operators $\mathbf{a}_{J M}^{c \lambda}(k)$ and $\mathbf{a}_{J M}^{c \lambda+}(k)$

$$
C \mathbf{a}_{J M}^{c \lambda}(k) C^{-1}=-\mathbf{a}_{J M}^{c \lambda}(k), C \mathbf{a}_{J M}^{c \lambda+}(k) C^{-1}=-\mathbf{a}_{J M}^{c \lambda+}(k),
$$

where $\lambda=S, M, E, L$.

Now let us turn to the angular momentum representation of spinor fields. As shown in Appendix, this representation may be set up by means of the spherical Dirac spinors. These spinors are shown in the following.

$$
\begin{gathered}
u_{J M}^{\sigma}(p \vec{x})=\left(\begin{array}{c}
\sqrt{\frac{\varepsilon+m}{2 \varepsilon}} u_{J}^{\sigma}(p r) \Omega_{J M}^{\sigma}(\widehat{x}) \\
-\sqrt{\frac{\varepsilon-m}{2 \varepsilon}} u_{J}^{-\sigma}(p r) \Omega_{J M}^{-\sigma}(\widehat{x})
\end{array}\right), \\
v_{J M}^{\sigma}(p \vec{x})=(-1)^{J+M+\sigma}\left(\begin{array}{c}
\sqrt{\frac{\varepsilon-m}{2 E}} u_{J}^{\sigma}(p r) \Omega_{J-M}^{-\sigma}(\widehat{x}) \\
\sqrt{\frac{\varepsilon+m}{2 \varepsilon}} u_{J}^{\sigma}(p r) \Omega_{J-M}^{\sigma}(\widehat{x})
\end{array}\right),
\end{gathered}
$$

where $J M$ denote the total angular momentum and its third component of a free fermion, $\sigma= \pm 1, \varepsilon=\sqrt{p^{2}+m^{2}}$ is the energy of the fermion in which $m$ is the mass and $\vec{p}$ is the momentum, $p=|\vec{p}|, \Omega_{J M}^{\sigma}(\widehat{x})$ is the spherical harmonic spinor defined as 


$$
\Omega_{J M}^{\sigma}(\widehat{x})=\left(\begin{array}{c}
\sigma \sqrt{\frac{J+\sigma\left(M-\frac{1}{2}\right)+\frac{1}{2}}{2 J-l+1}} Y_{J-\frac{\sigma}{2}, M-\frac{1}{2}}(\widehat{x}) \\
\sqrt{\frac{J-\sigma\left(M+\frac{1}{2}\right)+\frac{1}{2}}{2 J-\sigma+1}} Y_{J-\frac{\sigma}{2}, M+\frac{1}{2}}(\widehat{x})
\end{array}\right)
$$

and $u_{J}^{\sigma}(p r)$ is defined by

$$
u_{J}^{\sigma}(p r)=i^{J-\frac{\sigma}{2}} \sqrt{\frac{2}{\pi}} p j_{J-\frac{\sigma}{2}}(p r) .
$$

The spinors $u_{J M}^{\sigma}(p \vec{x})$ and $v_{J M}^{\sigma}(p \vec{x})$ are the eigenstates of total angular momentum for a fermion. They respectively obey the free Dirac equations of positive energy state and negative energy state. Based on the above expressions of the spherical Dirac spinors, it is easy to prove the following orthonormality and completeness relations

$$
\begin{aligned}
& \int d^{3} x u_{J M}^{\sigma^{+}}(p \vec{x}) u_{J^{\prime} M^{\prime}}^{\sigma}\left(p^{\prime} \vec{x}\right)=\delta\left(p-p^{\prime}\right) \delta_{J J^{\prime}} \delta_{\sigma \sigma^{\prime}} \delta_{M M^{\prime}}, \\
& \int d^{3} x v_{J M}^{\sigma^{+}}(p \vec{x}) v_{J^{\prime} M^{\prime}}^{\sigma^{\prime}}\left(p^{\prime} \vec{x}\right)=\delta\left(p-p^{\prime}\right) \delta_{J J^{\prime}} \delta_{\sigma \sigma^{\prime}} \delta_{M M^{\prime}}, \\
& \int d^{3} x u_{J M}^{\sigma^{+}}(p \vec{x}) v_{J^{\prime} M^{\prime}}^{\sigma^{\prime}}\left(p^{\prime} \vec{x}\right)=\int d^{3} x v_{J M}^{\sigma^{+}}(p \vec{x}) u_{J^{\prime} M^{\prime}}^{\sigma^{\prime}}\left(p^{\prime} \vec{x}\right)=0, \\
& \sum_{J M \sigma} \int_{0}^{\infty} d p\left[u_{J M}^{\sigma}(p \vec{x}) u_{J M}^{\sigma^{+}}\left(p \vec{x}^{\prime}\right)+v_{J M}^{\sigma}(p \vec{x}) v_{J M}^{\sigma^{+}}\left(p \vec{x}^{\prime}\right)\right]=\delta^{3}\left(\vec{x}-\vec{x}^{\prime}\right) .
\end{aligned}
$$

Clearly, the free quark field operators may be expanded in terms of the spherical Dirac spinors

$$
\begin{aligned}
& \psi(x)=\sum_{s \sigma J M} \int_{0}^{\infty} d p\left[\mathbf{b}_{J M}^{s \sigma}(p) u_{J M}^{\sigma}(p \vec{x}) e^{-i \varepsilon t}+\mathbf{d}_{J M}^{s \sigma^{+}}(p) v_{J M}^{\sigma}(p \vec{x}) e^{i \varepsilon t}\right], \\
& \bar{\psi}(x)=\sum_{s \sigma J M} \int_{0}^{\infty} d p\left[\mathbf{b}_{J M}^{s \sigma^{+}}(p) \bar{u}_{J M}^{\sigma}(p \vec{x}) e^{i \varepsilon t}+\mathbf{d}_{J M}^{s \sigma}(p) \bar{v}_{J M}^{\sigma}(p \vec{x}) e^{-i \varepsilon t}\right],
\end{aligned}
$$

where $s=(c, f), c$ and $f$ are the color and flavor indices for a quark, $\bar{u}_{J M}^{\sigma}(p \vec{x})=u_{J M}^{\sigma}(p \vec{x})^{+} \gamma_{0}, \bar{v}_{J M}^{\sigma}(p \vec{x})=$ $v_{J M}^{\sigma}(p \vec{x})^{+} \gamma_{0}$ and $\mathbf{b}_{J M}^{s \sigma^{+}}(p), \mathbf{d}_{J M}^{s \sigma^{+}}(p)$ and $\mathbf{b}_{J M}^{s \sigma}(p), \mathbf{d}_{J M}^{s \sigma}(p)$ are the creation and annihilation operators. These operators satisfy the following anticommutation relations

$$
\begin{aligned}
& \left\{\mathbf{b}_{J M}^{s \sigma}(p), \mathbf{b}_{J^{\prime} M^{\prime}}^{s^{\prime} \sigma^{\prime}+}\left(p^{\prime}\right)\right\}=\delta\left(p-p^{\prime}\right) \delta_{s s^{\prime}} \delta_{\sigma \sigma^{\prime}} \delta_{J J^{\prime}} \delta_{M M^{\prime}} \\
& \left\{\mathbf{d}_{J M}^{s \sigma}(p), \mathbf{d}_{J^{\prime} M^{\prime}}^{s^{\prime} \sigma^{\prime}+}\left(p^{\prime}\right)\right\}=\delta\left(p-p^{\prime}\right) \delta_{s s^{\prime}} \delta_{\sigma \sigma^{\prime}} \delta_{J J^{\prime}} \delta_{M M^{\prime}}
\end{aligned}
$$

with the other anticommutators being zero.

From the space inversion transformation of the quark field [46]

$$
P \psi(\vec{x}, t) P^{-1}=\eta_{P} \gamma^{0} \psi(-\vec{x}, t)
$$

and the relations

$$
\begin{aligned}
\gamma^{0} u_{J M}^{\sigma}(-p \vec{x}) & =(-1)^{J-\frac{\sigma}{2}} u_{J M}^{\sigma}(p \vec{x}), \gamma^{0} v_{J M}^{\sigma}(-p \vec{x}) \\
& =(-1)^{J-\frac{\sigma}{2}+1} v_{J M}^{\sigma}(p \vec{x}),
\end{aligned}
$$

it is easy to find from Eqs.(3.27) and (3.28) the parity of the quark operators such that

$$
\begin{aligned}
& P \mathbf{b}_{l m}^{s \lambda}(p) P^{-1}=\eta_{P}(-1)^{J-\frac{\sigma}{2}} \mathbf{b}_{l m}^{s \lambda}(p), \\
& P \mathbf{d}_{l m}^{s \lambda}(p) P^{-1}=\eta_{P}(-1)^{J+\frac{\sigma}{2}+1} \mathbf{d}_{l m}^{s \lambda}(p), \\
& P \mathbf{b}_{l m}^{s \lambda^{+}}(p) P^{-1}=\eta_{P}(-1)^{J-\frac{\sigma}{2}} \mathbf{b}_{l m}^{s \lambda^{+}}(p), \\
& P \mathbf{d}_{l m}^{s \lambda^{+}}(p) P^{-1}=\eta_{P}(-1)^{J+\frac{\sigma}{2}+1} \mathbf{d}_{l m}^{s \lambda^{+}}(p),
\end{aligned}
$$

where the phase factor $\eta_{P}$ usually is chosen to be $\eta_{P}=1$. By noticing the charge conjugation transformations [46]

$$
\mathcal{B} \psi(x) \mathcal{B}^{-1}=\eta_{C} C \bar{\psi}(x)
$$

where $C=i \gamma^{2} \gamma^{0}$ and

$$
v_{J M}^{\sigma}(p \vec{x})=C \bar{u}_{J M}^{\sigma}(p \vec{x})^{T}, u_{J M}^{\sigma}(p \vec{x})=C \bar{v}_{J M}^{\sigma}(p \vec{x})^{T}
$$


the $C$-parities of the creation and annihilation operators are easily found from Eqs.(3.27) and (3.28)

$$
\begin{aligned}
& \mathcal{B} b_{l m}^{s \lambda}(p) \mathcal{B}^{-1}=\eta_{C} d_{l m}^{s \lambda}(p), \mathcal{B} d_{l m}^{s \lambda}(p) \mathcal{B}^{-1}=\eta_{C} b_{l m}^{s \lambda}(p), \\
& \mathcal{B} b_{l m}^{s \lambda^{+}}(p) \mathcal{B}^{-1}=\eta_{C} d_{l m}^{s \lambda^{+}}(p), \mathcal{B} d_{l m}^{s \lambda^{+}}(p) \mathcal{B}^{-1}=\eta_{C} b_{l m}^{s \lambda^{+}}(p) .
\end{aligned}
$$

For the ghost fields, considering their scalar character, we can write their expressions in the angular momentum representation as follows

$$
\begin{aligned}
& \mathbf{C}^{a}(x)=\sum_{J M} \int_{0}^{\infty} \frac{d k}{\sqrt{2 \omega}}\left[\mathbf{c}_{J M}^{a}(k) A_{J M}^{s}(k \vec{x}) e^{-i \omega t}+\mathbf{d}_{J M}^{a^{+}}(k) A_{J M}^{s^{*}}(k \vec{x}) e^{i \omega t}\right], \\
& \overline{\mathbf{C}}^{a}(x)=\sum_{J M} \int_{0}^{\infty} \frac{d k}{\sqrt{2 \omega}}\left[\mathbf{d}_{J M}^{a}(k) A_{J M}^{s}(k \vec{x}) e^{-i \omega t}+\mathbf{c}_{J M}^{a^{+}}(k) A_{J M}^{s^{*}}(k \vec{x}) e^{i \omega t}\right] .
\end{aligned}
$$

The anticommutation relations for the ghost particle operators in the above are

$$
\begin{aligned}
\left\{\mathbf{c}_{J M}^{a}(k), \mathbf{c}_{J^{\prime} M^{\prime}}^{a^{\prime}+}\left(k^{\prime}\right)\right\} & =\delta_{a a^{\prime}} \delta_{J J^{\prime}} \delta_{M M^{\prime}} \delta\left(k-k^{\prime}\right), \\
\left\{\mathbf{d}_{J M}^{a}(k), \mathbf{d}_{J^{\prime} M^{\prime}}^{a^{\prime}+}\left(k^{\prime}\right)\right\} & =\delta_{a a^{\prime}} \delta_{J J^{\prime}} \delta_{M M^{\prime}} \delta\left(k-k^{\prime}\right),
\end{aligned}
$$

with the other anticommutators being vanishing.

\section{QCD HAMILTONIAN IN ANGULAR MOMENTUM REPRESENTATION}

In this section, we are devoted to discussing the QCD Hamiltonian in the angular momentum representation. By virtue of the expansions given in Eqs.(3.14), (3.15), (3.27), (3.28), (3.36) and (3.37), it is not difficult to formulate the Hamiltonian derived from the Lorentz-covariant Lagrangian written in Eqs.(2.30) and (2.31). However, due to presence of the field $A^{0}(x)$, the expression of the Hamiltonian is rather complicated. Considering that the field $A^{0}(x)$ is unphysical and will eventually be eliminated from the S-matrix and the B-S equation by the ghost fields as ensured by the unitarity of the theory [45], for the sake of simplifying the representation of Hamiltonian, we would rather to start with the Lagrangian obtained from the Lagrangian in Eqs.(2.30) and (2.31) by setting the unphysical fields $A^{0}(x), \bar{C}^{a}(x)$ and $C^{a}(x)$ to vanish, as was commonly done in the lattice gauge calculations [25-30] and similarly done in Ref.[23] where a massive QCD Lagrangian taken in the temporal gauge was used for calculating the glueball spectrum in the framework of B-S equation. The Lagrangian we start with is

$$
\mathcal{L}=\mathcal{L}_{0}+\mathcal{L}_{I},
$$

where

$$
\begin{aligned}
\mathcal{L}_{0} & =-\frac{1}{2} A_{i}^{a}\left(\square+\mu^{2}\right) A_{i}^{a}+\bar{\psi}\left(i \gamma^{\mu} \partial_{\mu}-m\right) \psi \\
& =\frac{1}{2}\left(\dot{A}_{k}^{a}\right)^{2}+\frac{1}{2} A_{i}^{a}\left(\nabla^{2}-\mu^{2}\right) A_{i}^{a}+\bar{\psi}\left(i \gamma^{\mu} \partial_{\mu}-m\right) \psi
\end{aligned}
$$

and

$$
\begin{aligned}
\mathcal{L}_{I}= & -\frac{1}{2} g f^{a b c}\left(\partial_{i} A_{j}^{a}-\partial_{j} A_{i}^{a}\right) A_{i}^{b} A_{j}^{c} \\
& -\frac{1}{4} g^{2} f^{a b c} f^{a d e} A_{i}^{b} A_{j}^{c} A_{i}^{d} A_{j}^{e}-\bar{\psi} \gamma_{i} T^{a} \psi A_{i}^{a} .
\end{aligned}
$$

By making use of the canonical variables conjugate to $A_{k}^{a}, \Psi$ and $\bar{\Psi}$ which are defined by

$$
\begin{aligned}
& \Pi_{k}^{a}=\frac{\partial \mathcal{L}}{\partial \dot{A_{k}^{a}}}=A_{k}^{a}, \\
& \Pi_{\psi}=\frac{\partial \mathcal{L}}{\partial \dot{\psi}}=i \bar{\psi} \gamma^{0}, \\
& \Pi_{\bar{\psi}}=\frac{\partial \mathcal{L}}{\partial \dot{\bar{\psi}}}=0,
\end{aligned}
$$


we can write the Hamiltonian density as

$$
\begin{aligned}
\mathcal{H} & =\Pi_{k}^{a} \dot{A}_{k}^{a}+\Pi_{\psi} \dot{\psi}-\mathcal{L} \\
& =\mathcal{H}_{0}+\mathcal{H}_{I}
\end{aligned}
$$

where

$$
\mathcal{H}_{0}=\frac{1}{2}\left(\dot{A}_{k}^{a}\right)^{2}-\frac{1}{2} A_{i}^{a}\left(\nabla^{2}-\mu^{2}\right) A_{i}^{a}+\bar{\psi}(\vec{\alpha} \cdot \nabla+\beta m) \psi
$$

and

$$
\begin{aligned}
\mathcal{H}_{I}= & -\frac{g}{2} f^{a b c}\left(\overrightarrow{A^{a}} \times \overrightarrow{A^{b}}\right) \cdot\left(\nabla \times \overrightarrow{A^{c}}\right) \\
& +\frac{1}{8} g^{2} f^{a b e} f^{c d e}\left(\overrightarrow{A^{a}} \times \overrightarrow{A^{b}}\right) \cdot\left(\overrightarrow{A^{c}} \times \overrightarrow{A^{d}}\right) \\
& +g \bar{\psi} T^{a} \vec{\gamma} \cdot \overrightarrow{A^{a}} \psi .
\end{aligned}
$$

The first two terms in Eq.(4.6) denote the energy of free gluon fields. The second term gives the energy of free quark fields. In the interaction Hamiltonian density (4.7), the first, second and third terms represent the gluon three-line vertex, the gluon four-line vertex and quark-gluon vertex respectively. It should be noted that the above Hamiltonian includes not only the transverse field $\vec{A}_{T}$, but also the longitudinal field $\vec{A}_{L}$ whose presence can be seen from the constraint condition in Eq. (2.11) because in the case of $A^{0}=0$ and $\alpha=1$, the condition becomes $\nabla \cdot \overrightarrow{A_{L}^{c}}=-\lambda \neq 0$. This is different from the theory proposed in Ref.[23] where only the transverse field exists.

Now let us derive the expression of the Hamiltonian given by the Hamiltonian density shown above in the angular momentum representation. First, we derive the expression of the free Hamiltonian. On substituting Eqs.(3.14),(3.15),(3.27),(3.28) into Eq.(4.6), one may get

$$
\begin{aligned}
H_{0}= & \int d^{3} x \mathcal{H}_{0} \\
= & \sum_{c \lambda J M} \int_{0}^{\infty} d k \omega(\vec{k}) \mathbf{a}_{J M}^{c \lambda^{+}}(k) \mathbf{a}_{J M}^{c \lambda}(k) \\
& +\sum_{s \sigma l m} \int_{0}^{\infty} d p \varepsilon(\vec{p})\left[\mathbf{b}_{J M}^{s \sigma^{+}}(p) \mathbf{b}_{J M}^{s \sigma}(p)+\mathbf{d}_{J M}^{s \sigma^{+}}(p) \mathbf{d}_{J M}^{s \sigma}(p)\right] .
\end{aligned}
$$

In later derivations, it is convenient to introduce compact notations for various indices so as to simplify the expressions of the operators and formulas. For the gluon field, we define

$$
\begin{gathered}
\mathbf{a}_{J M}^{c \lambda \xi}(k)=\left\{\begin{array}{l}
\mathbf{a}_{J M}^{c \lambda}(k) \text { if } \xi=1 \\
\mathbf{a}_{J M}^{c \lambda^{+}}(k) \text { if } \xi=-1
\end{array},\right. \\
\vec{A}_{J M}^{\lambda \xi}(k \vec{x})=\left\{\begin{array}{l}
\vec{A}_{J M}^{\lambda}(k \vec{x}) \text { if } \xi=1 \\
\vec{A}_{J M}^{\lambda^{*}}(k \vec{x}) \text { if } \xi=-1
\end{array},\right.
\end{gathered}
$$

then, the commutators in Eqs.(3.16) and (3.17) can be unified to write as

$$
\left[\mathbf{a}_{J M}^{c \lambda \xi}(k), \mathbf{a}_{J^{\prime} M^{\prime}}^{c^{\prime} \lambda^{\prime} \xi^{\prime}}\left(k^{\prime}\right)\right]=\delta\left(k-k^{\prime}\right) \delta_{c c^{\prime}} \delta_{\lambda \lambda^{\prime}} \delta_{J J^{\prime}} \delta_{M M^{\prime}} \sin \left[\left(\xi-\xi^{\prime}\right) \frac{\pi}{4}\right]
$$

Furthermore, we define $\alpha \equiv(c, \lambda, J, M, k, \xi)$ in which the $\xi$ will be written as $\xi_{\alpha}$ later on. In this notation, the commutator in Eq.(4.11) will be represented as

$$
\left[\mathbf{a}_{\alpha}, \mathbf{a}_{\beta}\right]=\triangle_{\alpha \beta},
$$

where

$$
\triangle_{\alpha \beta}=\delta\left(k-k^{\prime}\right) \delta_{c c^{\prime}} \delta_{\lambda \lambda^{\prime}} \delta_{J J^{\prime}} \delta_{M M^{\prime}} \sin \left[\left(\xi-\xi^{\prime}\right) \frac{\pi}{4}\right]
$$


and the expansion in Eq.(3.14) becomes

$$
\overrightarrow{A^{c}}(x)=\sum_{\alpha} \mathbf{a}_{\alpha} \vec{A}_{\alpha}(k \vec{x}) e^{-i \xi_{\alpha} \omega_{\alpha} t}
$$

where

$$
\begin{gathered}
\sum_{\alpha} \equiv \sum_{c \lambda l m} \int_{0}^{\infty} d k, \omega_{\alpha}=\sqrt{k^{2}+\mu^{2}} \\
\vec{A}_{\alpha}(k \vec{x})=\frac{1}{\sqrt{2 \omega_{\alpha}}} \vec{A}_{J M}^{\lambda \xi}(k \vec{x}) .
\end{gathered}
$$

For the quark field, with the definition

$$
\begin{gathered}
\mathbf{c}_{l m}^{s \sigma \eta}(p)=\left\{\begin{array}{ll}
\mathbf{b}_{l m}^{s \sigma}(p) & \text { if } \eta=1 \\
\mathbf{d}_{l m}^{s \sigma}(p) & \text { if } \eta=-1
\end{array},\right. \\
w_{l m}^{\sigma \eta}(p \vec{x})=\left\{\begin{array}{ll}
u_{l m}^{\sigma}(p \vec{x}) & \text { if } \eta=1 \\
v_{l m}^{\sigma}(p \vec{x}) & \text { if } \eta=-1
\end{array},\right.
\end{gathered}
$$

the anticommutators in Eq.(3.29) becomes

$$
\left\{\mathbf{c}_{J M}^{s \sigma \eta}(p), \mathbf{c}_{J^{\prime} M^{\prime}}^{s^{\prime} \sigma^{\prime} \eta^{\prime}+}\left(p^{\prime}\right)\right\}=\delta\left(p-p^{\prime}\right) \delta_{s s^{\prime}} \delta_{\sigma \sigma^{\prime}} \delta_{J J^{\prime}} \delta_{M M^{\prime}} \delta_{\eta \eta^{\prime}} .
$$

When we set $\alpha=(s, \sigma, l, m, p, \eta)$ in which the $\eta$ will be written as $\eta_{\alpha}$ later on, the above anticommutator can be simply written as

$$
\left\{\mathbf{c}_{\alpha}, \mathbf{c}_{\beta}^{+}\right\}=\delta_{\alpha \beta},
$$

where

$$
\delta_{\alpha \beta} \equiv \delta\left(p-p^{\prime}\right) \delta_{s s^{\prime}} \delta_{\sigma \sigma^{\prime}} \delta_{J J^{\prime}} \delta_{M M^{\prime}} \delta_{\eta \eta^{\prime}},
$$

and the expansions in Eqs.(3.27) and (3.28) will be represented by

$$
\begin{aligned}
& \psi(x)=\sum_{\alpha} \mathbf{c}_{\alpha} w_{\alpha}(p \vec{x}) e^{-i \xi_{\alpha} \varepsilon_{\alpha} t}, \\
& \bar{\psi}(x)=\sum_{\alpha} \mathbf{c}_{\alpha}^{+} \bar{w}_{\alpha}(p \vec{x}) e^{i \xi_{\alpha} \varepsilon_{\alpha} t},
\end{aligned}
$$

where

$$
\sum_{\alpha} \equiv \sum_{s \lambda l m} \int_{0}^{\infty} d p, \quad \varepsilon_{\alpha}=\sqrt{p^{2}+m^{2}} .
$$

In view of the notations defined above, the free Hamiltonian will compactly be rewritten as follows

$$
\begin{gathered}
H^{0}=H_{g}^{0}+H_{q}^{0}, \\
H_{g}^{0}=\frac{1}{2} \sum_{\alpha \beta} \omega_{\alpha \beta}: \mathbf{a}_{\alpha} \mathbf{a}_{\beta}: \\
H_{q}^{0}=\sum_{\alpha} \xi_{\alpha} \varepsilon_{\alpha}: \mathbf{c}_{\alpha}^{+} \mathbf{c}_{\alpha}:
\end{gathered}
$$

where the symbol :: denotes the normal product and

$$
\omega_{\alpha \beta} \equiv \omega(k) \delta\left(k-k^{\prime}\right) \delta_{c c^{\prime}} \delta_{\lambda \lambda^{\prime}} \delta_{J J^{\prime}} \delta_{M M^{\prime}}\left(1-\delta_{\xi \xi^{\prime}}\right) .
$$


Next, we derive the expression of the interaction Hamiltonian in the angular momentum representation. In doing this, It is helpful to use the transformations for an operator ( a Hamiltonian, a field operator, a creation or annihilation operator) among the Heisenberg picture, the Schrödinger picture and the interaction picture

$$
\mathbf{F}_{H}(t)=e^{i H t} \mathbf{F}_{s} e^{-i H t}=e^{i H t} e^{-H_{0} t} \mathbf{F}_{I}(t) e^{i H_{0} t} e^{-i H t},
$$

where $\mathbf{F}_{H}(t), \mathbf{F}_{s}$ and $\mathbf{F}_{I}(t)$ stand for the operators given in the the Heisenberg, Schrödinger and interaction pictures. From Eq.(4.28), it is clearly seen that $\mathbf{F}_{s}=\mathbf{F}_{H}(0)=\mathbf{F}_{I}(0)$ which is independent of time. According to the relation in Eq.(4.28), we only need to write out the interaction Hamiltonian in the Schrödinger picture (or in the interaction picture). In this picture, on inserting the expressions in Eqs.(4.14) and (4.22) into Eq.(4.7), we obtain

$$
\begin{aligned}
H_{I}(0) & =\int d^{3} x \mathcal{H}_{I}(\vec{x}, 0) \\
& =\sum_{\alpha \beta \gamma} A(\alpha \beta \gamma): \mathbf{a}_{\alpha} \mathbf{a}_{\beta} \mathbf{a}_{\gamma}: \\
+\sum_{\alpha \beta \gamma \delta} B(\alpha \beta \gamma \delta) & : \mathbf{a}_{\alpha} \mathbf{a}_{\beta} \mathbf{a}_{\gamma} \mathbf{a}_{\delta}: \\
+\sum_{\alpha \beta \gamma} C(\alpha \beta \gamma) & : \mathbf{c}_{\alpha}^{+} \mathbf{c}_{\beta} \mathbf{a}_{\gamma}:
\end{aligned}
$$

where

$$
\begin{gathered}
A(\alpha \beta \gamma)=-\frac{g}{2} f^{a b c} \int d^{3} x\left(\overrightarrow{A_{\alpha}} \times \overrightarrow{A_{\beta}}\right) \cdot\left(\nabla \times \overrightarrow{A_{\gamma}}\right), \\
B(\alpha \beta \gamma \delta)=\frac{1}{8} g^{2} f^{a b e} f^{c d e} \int d^{3} x\left(\overrightarrow{A_{\alpha}} \times \overrightarrow{A_{\beta}}\right) \cdot\left(\overrightarrow{A_{\gamma}} \times \overrightarrow{A_{\delta}}\right)
\end{gathered}
$$

and

$$
C(\alpha \beta \gamma)=g \int d^{3} x \bar{w}_{\alpha} T^{c} \vec{\gamma} w_{\beta} \cdot \overrightarrow{A_{\gamma}} .
$$

By making use of the expressions of $\overrightarrow{A_{\alpha}}, \bar{w}_{\alpha}$ and $w_{\beta}$, Completing the integrals over $\vec{x}$ and applying the formulas of angular momentum couplings, one may derive explicit expressions of the coefficients $A(\alpha \beta \gamma), B(\alpha \beta \gamma \delta)$ and $C(\alpha \beta \gamma)$. The expressions of $A(\alpha \beta \gamma)$ and $B(\alpha \beta \gamma \delta)$ will be given in the next paper. As for the coefficient $C(\alpha \beta \gamma)$,its expression will be presented later in other publication.

\section{THREE-DIMENSIONAL RELATIVISTIC EQUATION FOR TWO GLUON BOUND STATES}

The aim of this section is to derive a rigorous three-dimensional relativistic equation satisfied by two-gluon bound states. This equation may be derived from the equation satisfied by the following gluon two-time Green's functions defined in the Heisenberg picture. These Green's functions include

$$
\begin{aligned}
& G\left(\alpha^{+} \beta^{+} ; \gamma^{-} \delta^{-} ; t_{1}-t_{2}\right)=\left\langle 0^{+}\left|T\left\{\mathbf{a}_{\alpha}\left(t_{1}\right) \mathbf{a}_{\beta}\left(t_{1}\right) \mathbf{a}_{\gamma}^{+}\left(t_{2}\right) \mathbf{a}_{\delta}^{+}\left(t_{2}\right)\right\}\right| 0^{-}\right\rangle, \\
& G\left(\alpha^{-} \beta^{-} ; \gamma^{+} \delta^{+} ; t_{1}-t_{2}\right)=\left\langle 0^{+}\left|T\left\{\mathbf{a}_{\alpha}^{+}\left(t_{1}\right) \mathbf{a}_{\beta}^{+}\left(t_{1}\right) \mathbf{a}_{\gamma}\left(t_{2}\right) \mathbf{a}_{\delta}\left(t_{2}\right)\right\}\right| 0^{-}\right\rangle, \\
& G\left(\alpha^{-} \beta^{+} ; \gamma^{-} \delta^{+} ; t_{1}-t_{2}\right)=\left\langle 0^{+}\left|T\left\{\mathbf{a}_{\alpha}^{+}\left(t_{1}\right) \mathbf{a}_{\beta}\left(t_{1}\right) \mathbf{a}_{\gamma}^{+}\left(t_{2}\right) \mathbf{a}_{\delta}\left(t_{2}\right)\right\}\right| 0^{-}\right\rangle, \\
& G\left(\alpha^{+} \beta^{-} ; \gamma^{+} \delta^{-} ; t_{1}-t_{2}\right)=\left\langle 0^{+}\left|T\left\{\mathbf{a}_{\alpha}\left(t_{1}\right) \mathbf{a}_{\beta}^{+}\left(t_{1}\right) \mathbf{a}_{\gamma}\left(t_{2}\right) \mathbf{a}_{\delta}^{+}\left(t_{2}\right)\right\}\right| 0^{-}\right\rangle,
\end{aligned}
$$

where $T$ symbolizes the time-ordered product and $\left|0^{ \pm}\right\rangle$denote the physical vacuum states defined in Heisenberg picture. It should be noted that the creation and annihilation operators in Eq.(5.1) are those defined in Eq.(3.15). For later convenience, we will use the notation defined in Eq.(4.9) for the gluon operators. With this notation, instead of the above Green's functions, we may start, in a consistent way, from the following Green's function defined by

$$
G\left(\alpha \beta ; \gamma \delta ; t_{1}-t_{2}\right)=\left\langle 0^{+}\left|T\left\{\mathbf{a}_{\alpha}\left(t_{1}\right) \mathbf{a}_{\beta}\left(t_{1}\right) \mathbf{a}_{\gamma}\left(t_{2}\right) \mathbf{a}_{\delta}\left(t_{2}\right)\right\}\right| 0^{-}\right\rangle,
$$


where $\mathbf{a}_{\alpha}$ can be a creation operator or an annihilation one.

On differentiating Eq.(5.2) with respect to time $t_{1}$, it is found that

$$
\begin{aligned}
& i \frac{\partial}{\partial t_{1}} G\left(\alpha \beta ; \gamma \delta ; t_{1}-t_{2}\right)=i \delta\left(t_{1}-t_{2}\right) S(\alpha \beta, \gamma \delta) \\
& +\left\langle 0^{+}\right| T\left\{\left[\left(i \frac{\partial}{\partial t_{1}} \mathbf{a}_{\alpha}\left(t_{1}\right)\right) \mathbf{a}_{\beta}\left(t_{1}\right)\right.\right. \\
& \left.\left.+\mathbf{a}_{\alpha}\left(t_{1}\right)\left(i \frac{\partial}{\partial t_{1}} \mathbf{a}_{\beta}\left(t_{1}\right)\right)\right] \mathbf{a}_{\gamma}\left(t_{2}\right) \mathbf{a}_{\delta}\left(t_{2}\right)\right\}\left|0^{-}\right\rangle,
\end{aligned}
$$

where

$$
\begin{aligned}
& S(\alpha \beta, \gamma \delta)=\left\langle 0^{+}\left|\left[\mathbf{a}_{\alpha}\left(t_{1}\right) \mathbf{a}_{\beta}\left(t_{1}\right), \mathbf{a}_{\gamma}\left(t_{2}\right) \mathbf{a}_{\delta}\left(t_{2}\right)\right]\right| 0^{-}\right\rangle \\
& =\left\langle 0^{+}\left|\left[\mathbf{a}_{\alpha} \mathbf{a}_{\beta}, \mathbf{a}_{\gamma} \mathbf{a}_{\delta}\right]\right| 0^{-}\right\rangle
\end{aligned}
$$

is time-independent owing to the time displacement shown in Eq.(4.28) and the restriction of $\delta\left(t_{1}-t_{2}\right)$. According to the relation in Eq.(4.28), we have

$$
i \frac{\partial}{\partial t} \mathbf{a}_{\alpha}(t)=e^{i H t}\left[\mathbf{a}_{\alpha}, H\right] e^{-i H t}
$$

Since $\mathbf{a}_{\alpha}$ in the commutator is independent of time, we may use the Hamiltonian given in Eqs.(4.24)-(4.26) and (4.29) and the commutation relation in Eq.(4.12) to compute the commutator $\left[\mathbf{a}_{\alpha}, H\right]$. When the result is substituted in Eq.(5.5), we obtain

$$
\begin{aligned}
& i \frac{\partial}{\partial t_{1}} \mathbf{a}_{\alpha}\left(t_{1}\right)=\sum_{\rho \sigma} \omega_{\rho \sigma} \Delta_{\alpha \sigma} \mathbf{a}_{\rho}\left(t_{1}\right)+\sum_{\rho \sigma \tau} f_{1}(\rho \sigma \tau) \Delta_{\alpha \tau}: \mathbf{a}_{\rho}\left(t_{1}\right) \mathbf{a}_{\sigma}\left(t_{1}\right): \\
& +\sum_{\rho \sigma \tau \lambda} f_{2}(\rho \sigma \tau \lambda) \Delta_{\alpha \lambda}: \mathbf{a}_{\rho}\left(t_{1}\right) \mathbf{a}_{\sigma}\left(t_{1}\right) \mathbf{a}_{\tau}\left(t_{1}\right): \\
& +\sum_{\rho \sigma \tau} f_{3}(\rho \sigma \tau) \Delta_{\alpha \tau}: \mathbf{c}_{\rho}^{+}\left(t_{1}\right) \mathbf{c}_{\sigma}\left(t_{1}\right):
\end{aligned}
$$

where $\Delta_{\alpha \sigma}$ was defined in Eq.(4.13) and the coefficients $f_{i}$ are defined by

$$
\begin{aligned}
& f_{1}(\rho \sigma \tau)=A(\rho \sigma \tau)+A(\rho \tau \sigma)+A(\tau \rho \sigma), \\
& f_{2}(\rho \sigma \tau \lambda)=B(\rho \sigma \tau \lambda)+B(\rho \sigma \lambda \tau)+B(\rho \lambda \sigma \tau)+B(\lambda \rho \sigma \tau), \\
& f_{3}(\rho \sigma \tau)=C(\rho \sigma \tau) .
\end{aligned}
$$

Clearly, the expression of $i \frac{\partial}{\partial t_{1}} \mathbf{a}_{\beta}\left(t_{1}\right)$ may be written out from Eq.(5.6) by replacing $\alpha$ with $\beta$. Inserting this expression and that given in Eq.(5.6) into Eq.(5.3), one may find an equation of motion obeyed by the Green's function denoted in Eq.(5.2) such that

$$
\begin{aligned}
& i \frac{\partial}{\partial t_{1}} G\left(\alpha \beta ; \gamma \delta ; t_{1}-t_{2}\right)=i \delta\left(t_{1}-t_{2}\right) S(\alpha \beta, \gamma \delta) \\
& +\sum_{\rho \sigma \lambda} \omega_{\rho \sigma} \Delta_{\alpha \beta, \sigma \lambda} G\left(\rho \lambda ; \gamma \delta ; t_{1}-t_{2}\right) \\
& +\sum_{\rho \sigma \lambda} g_{1}(\alpha \beta ; \rho \sigma \lambda) G_{1}\left(\rho \sigma \lambda ; \gamma \delta ; t_{1}-t_{2}\right) \\
& +\sum_{\rho \sigma \tau \lambda} g_{2}(\alpha \beta ; \rho \sigma \tau \lambda) G_{2}\left(\rho \sigma \tau \lambda ; \gamma \delta ; t_{1}-t_{2}\right) \\
& +\sum_{\rho \sigma \lambda} g_{3}(\alpha \beta ; \rho \sigma \lambda) G_{3}\left(\rho \sigma \lambda ; \gamma \delta ; t_{1}-t_{2}\right),
\end{aligned}
$$

where

$$
\begin{gathered}
\Delta_{\alpha \beta, \sigma \lambda}=\Delta_{\alpha \sigma} \delta_{\beta \lambda}+\Delta_{\beta \sigma} \delta_{\alpha \lambda}, \\
g_{1}(\alpha \beta ; \rho \sigma \lambda)=\sum_{\tau} f_{1}(\rho \sigma \tau) \Delta_{\alpha \beta, \tau \lambda}, \\
g_{2}(\alpha \beta ; \rho \sigma \tau \lambda)=\sum_{\theta} f_{2}(\rho \sigma \tau \theta) \Delta_{\alpha \beta, \theta \lambda}, \\
g_{3}(\alpha \beta ; \rho \sigma \lambda)=\sum_{\tau} f_{3}(\rho \sigma \tau) \Delta_{\alpha \beta, \tau \lambda},
\end{gathered}
$$

and the Green functions $G_{i}$ are defined by

$$
\begin{aligned}
& G_{1}\left(\rho \sigma \lambda ; \gamma \delta ; t_{1}-t_{2}\right) \\
= & \left\langle 0^{+}\left|T\left\{: \mathbf{a}_{\rho}\left(t_{1}\right) \mathbf{a}_{\sigma}\left(t_{1}\right): \mathbf{a}_{\lambda}\left(t_{1}\right) \mathbf{a}_{\gamma}\left(t_{2}\right) \mathbf{a}_{\delta}\left(t_{2}\right)\right\}\right| 0^{-}\right\rangle,
\end{aligned}
$$




$$
\begin{aligned}
& G_{2}\left(\rho \sigma \tau \theta ; \gamma \delta ; t_{1}-t_{2}\right) \\
= & \left\langle 0^{+}\left|T\left\{: \mathbf{a}_{\rho}\left(t_{1}\right) \mathbf{a}_{\sigma}\left(t_{1}\right) \mathbf{a}_{\tau}\left(t_{1}\right): \mathbf{a}_{\theta}\left(t_{1}\right) \mathbf{a}_{\gamma}\left(t_{2}\right) \mathbf{a}_{\delta}\left(t_{2}\right)\right\}\right| 0^{-}\right\rangle, \\
& G_{3}\left(\rho \sigma \lambda ; \gamma \delta ; t_{1}-t_{2}\right) \\
= & \left\langle 0^{+}\left|T\left\{: \mathbf{c}_{\rho}^{+}\left(t_{1}\right) \mathbf{c}_{\sigma}\left(t_{1}\right): \mathbf{a}_{\lambda}\left(t_{1}\right) \mathbf{a}_{\gamma}\left(t_{2}\right) \mathbf{a}_{\delta}\left(t_{2}\right)\right\}\right| 0^{-}\right\rangle .
\end{aligned}
$$

By the Fourier transformation

$$
G_{i}(\alpha, \ldots, \delta ; \omega)=\frac{1}{i} \int_{-\infty}^{+\infty} d t e^{i \omega t} G_{i}(\alpha, \ldots, \delta ; t),
$$

the equation in Eq.(5.8) will be of the form in the energy representation

$$
\begin{aligned}
& \omega G(\alpha \beta ; \gamma \delta ; \omega)=S(\alpha \beta, \gamma \delta) \\
& +\sum_{\rho \sigma \lambda} \omega_{\rho \sigma} \Delta_{\alpha \beta, \sigma \lambda} G(\rho \lambda ; \gamma \delta ; \omega) \\
& +\sum_{\rho \sigma \lambda} g_{1}(\alpha \beta ; \rho \sigma \lambda) G_{1}(\rho \sigma \lambda ; \gamma \delta ; \omega) \\
& +\sum_{\rho \sigma \tau \lambda} g_{2}(\alpha \beta ; \rho \sigma \tau \lambda) G_{2}(\rho \sigma \tau \lambda ; \gamma \delta ; \omega) \\
& +\sum_{\rho \sigma \lambda} g_{3}(\alpha \beta ; \rho \sigma \lambda) G_{3}(\rho \sigma \lambda ; \gamma \delta ; \omega) .
\end{aligned}
$$

Let us look at the second term on the right hand side (RHS) of the above equation. From the definitions of $\omega_{\rho \sigma}$ and $\Delta_{\alpha \beta, \sigma \lambda}$ given in Eqs.(4.27), (5.9) and (4.13), we see, only when $\xi_{\rho} \neq \xi_{\sigma}, \omega_{\rho \sigma}$ and $\Delta_{\rho \sigma}$ are nonvanishing. When we define

$$
\omega_{\alpha}=\left\{\begin{array}{cc}
\omega(k) & \text { if } \xi_{\alpha}=+1 \\
-\omega(k) & \text { if } \xi_{\alpha}=-1
\end{array}\right.
$$

the second term mentioned above gives

$$
\sum_{\rho \sigma \lambda} \omega_{\rho \sigma} \Delta_{\alpha \beta, \sigma \lambda} G(\rho \lambda ; \gamma \delta ; \omega)=\left(\omega_{\alpha}+\omega_{\beta}\right) G(\alpha \beta ; \gamma \delta ; \omega) .
$$

Thus, Eq.(5.15) can be written as

$$
\begin{aligned}
& \left(\omega-\omega_{\alpha}-\omega_{\beta}\right) G(\alpha \beta ; \gamma \delta ; \omega) \\
& =S(\alpha \beta, \gamma \delta)+\sum_{\rho \sigma \lambda} g_{1}(\alpha \beta ; \rho \sigma \lambda) G_{1}(\rho \sigma \lambda ; \gamma \delta ; \omega) \\
& +\sum_{\rho \sigma \tau \lambda} g_{2}(\alpha \beta ; \rho \sigma \tau \lambda) G_{2}(\rho \sigma \tau \lambda ; \gamma \delta ; \omega) \\
& +\sum_{\rho \sigma \lambda} g_{3}(\alpha \beta ; \rho \sigma \lambda) G_{3}(\rho \sigma \lambda ; \gamma \delta ; \omega) .
\end{aligned}
$$

In order to obtain a closed equation, it is necessary (actually possible) to introduce effective interaction kernels $\Lambda_{i}$ in such a fashion

$$
\begin{aligned}
& \sum_{\rho \sigma \lambda} g_{1}(\alpha \beta ; \rho \sigma \lambda) G_{1}(\rho \sigma \lambda ; \gamma \delta ; \omega) \\
= & \sum_{\rho \sigma} \Lambda_{1}(\alpha \beta ; \rho \sigma ; \omega) G(\rho \sigma ; \gamma \delta ; \omega), \\
& \sum_{\rho \sigma \tau \lambda} g_{2}(\alpha \beta ; \rho \sigma \tau \lambda) G_{2}(\rho \sigma \tau \lambda ; \gamma \delta ; \omega) \\
= & \sum_{\rho \sigma} \Lambda_{2}(\alpha \beta ; \rho \sigma ; \omega) G(\rho \sigma ; \gamma \delta ; \omega),
\end{aligned}
$$




$$
\begin{aligned}
& \sum_{\rho \sigma \lambda} g_{3}(\alpha \beta ; \rho \sigma \lambda) G_{3}(\rho \sigma \lambda ; \gamma \delta ; \omega) \\
= & \sum_{\rho \sigma} \Lambda_{3}(\alpha \beta ; \rho \sigma ; \omega) G(\rho \sigma ; \gamma \delta ; \omega) .
\end{aligned}
$$

With the kernels defined above, Eq.(5.18) becomes

$$
\begin{aligned}
& \left(\omega-\omega_{\alpha}-\omega_{\beta}\right) G(\alpha \beta ; \gamma \delta ; \omega) \\
= & S(\alpha \beta, \gamma \delta)+\sum_{\rho \sigma} K(\alpha \beta ; \rho \sigma ; \omega) G(\rho \sigma ; \gamma \delta ; \omega),
\end{aligned}
$$

this is just the equation satisfied by the Green's function $G(\alpha \beta ; \gamma \delta ; \omega)$ in which

$$
K(\alpha \beta ; \rho \sigma ; \omega)=\sum_{i=1}^{3} \Lambda_{i}(\alpha \beta ; \rho \sigma ; \omega) .
$$

is the total interaction kernel.

The equation obeyed by glueball states may be derived from the above equation with the aid of the Lehmann representation of the Green's function. Suppose $\{|n\rangle\}$ form a complete set of glueball states, noticing the transformation in Eq.(4.28) and $H|n\rangle=E_{n}|n\rangle$, we can write

$$
\begin{aligned}
& G\left(\alpha \beta ; \gamma \delta ; t_{1}-t_{2}\right) \\
& =\sum_{n}\left\{\theta\left(t_{1}-t_{2}\right)\left\langle 0^{+}\left|\mathbf{a}_{\alpha} \mathbf{a}_{\beta}\right| n\right\rangle\left\langle n\left|\mathbf{a}_{\gamma} \mathbf{a}_{\delta}\right| 0^{-}\right\rangle e^{-i E_{n}\left(t_{1}-t_{2}\right)}\right. \\
& \left.+\theta\left(t_{2}-t_{1}\right)\left\langle 0^{+}\left|\mathbf{a}_{\gamma} \mathbf{a}_{\delta}\right| n\right\rangle\left\langle n\left|\mathbf{a}_{\alpha} \mathbf{a}_{\beta}\right| 0^{-}\right\rangle e^{-i E_{n}\left(t_{2}-t_{1}\right)}\right\} .
\end{aligned}
$$

Substituting in Eq.(5.24) the representation of the step function

$$
\theta(t)=\frac{i}{2 \pi} \int d q \frac{e^{-i q t}}{q+i \epsilon}
$$

and then performing a Fourier transformation with respect to time, we can get from Eq.(5.24) the Lehmann representation such that

$$
G(\alpha \beta ; \gamma \delta ; \omega)=\sum_{n}\left[\frac{\chi_{\alpha \beta}(n) \bar{\chi}_{\gamma \delta}(n)}{\omega-E_{n}+i \epsilon}-\frac{\chi_{\gamma \delta}(n) \bar{\chi}_{\alpha \beta}(n)}{\omega+E_{n}-i \epsilon}\right],
$$

where $E_{n}$ is the total energy of a glueball state and

$$
\chi_{\alpha \beta}(n)=\left\langle 0^{+}\left|\mathbf{a}_{\alpha} \mathbf{a}_{\beta}\right| n\right\rangle, \quad \bar{\chi}_{\alpha \beta}(n)=\left\langle n\left|\mathbf{a}_{\alpha} \mathbf{a}_{\beta}\right| 0^{-}\right\rangle
$$

are the B-S amplitudes describing the two-gluon bound states. On replacing the $G(\alpha \beta ; \gamma \delta ; \omega)$ in Eq.(5.22) with its Lehmann representation, then multiplying the both sides of Eq.(5.22) with $\omega-E_{n}$ and finally taking the limit $\omega \longrightarrow E_{n}$, we eventually arrive at

$$
\left(E_{n}-\omega_{\alpha}-\omega_{\beta}\right) \chi_{\alpha \beta}(n)=\sum_{\rho \sigma} K\left(\alpha \beta ; \rho \sigma ; E_{n}\right) \chi_{\rho \sigma}(n) .
$$

This is just the wanted three-dimensional relativistic equation for the two-gluon bound states. It should be pointed out that the above equation actually represents a set of coupled equations which may be written out by setting $\xi_{\alpha}$ in the index $\alpha$ and $\xi_{\beta}$ in the index $\beta$ to be \pm 1 .Each of the equations is manifestly of Schrödinger-type, i.e., a standard eigen-equation. In the next paper, these coupled equations will be reduced to an equivalent equation satisfied only by the B-S amplitude for which the two gluons are in the positive energy states.

\section{CLOSED EXPRESSION OF THE INTERACTION KERNEL}

In this section, we are devoted to derive the effective interaction kernel appearing in Eq.(5.28) and defined in Eq.(5.23), giving a closed expression of it. For this derivation, we need equations of motion which describe the 
evolution of the Green's function $G\left(\alpha \beta ; \gamma \delta ; t_{1}-t_{2}\right)$ and those shown in Eqs.(5.11)-(5.13) with time $t_{2}$. Taking the derivative of the Green's function in Eq.(5.2) with respect to $t_{2}$, we have

$$
\begin{aligned}
& i \frac{\partial}{\partial t_{2}} G\left(\alpha \beta ; \gamma \delta ; t_{1}-t_{2}\right)=-i \delta\left(t_{1}-t_{2}\right) S(\alpha \beta, \gamma \delta) \\
& +\left\langle 0^{+}\left|T\left\{\mathbf{a}_{\beta}\left(t_{1}\right) \mathbf{a}_{\beta}\left(t_{1}\right)\left[\left(i \frac{\partial}{\partial t_{2}} \mathbf{a}_{\gamma}\left(t_{2}\right)\right) \mathbf{a}_{\delta}\left(t_{2}\right)+\mathbf{a}_{\gamma}\left(t_{2}\right) i \frac{\partial}{\partial t_{2}} \mathbf{a}_{\delta}\left(t_{2}\right)\right]\right\}\right| 0^{-}\right\rangle,
\end{aligned}
$$

where

$$
\begin{aligned}
& i \frac{\partial}{\partial t_{2}} \mathbf{a}_{\gamma}\left(t_{2}\right)=\sum_{\rho \sigma} \omega_{\rho \sigma} \Delta_{\lambda \sigma} \mathbf{a}_{\rho}\left(t_{2}\right) \\
& +\sum_{\rho \sigma \tau} f_{1}(\rho \sigma \tau) \Delta_{\gamma \tau}: \mathbf{a}_{\rho}\left(t_{2}\right) \mathbf{a}_{\sigma}\left(t_{2}\right): \\
& +\sum_{\rho \sigma \tau \lambda} f_{2}(\rho \sigma \tau \lambda) \Delta_{\gamma \lambda}: \mathbf{a}_{\rho}\left(t_{2}\right) \mathbf{a}_{\sigma}\left(t_{2}\right) \mathbf{a}_{\tau}\left(t_{2}\right): \\
& +\sum_{\rho \sigma \tau} f_{3}(\rho \sigma \tau) \Delta_{\gamma \tau}: \mathbf{c}_{\rho}^{+}\left(t_{2}\right) \mathbf{c}_{\sigma}\left(t_{2}\right):
\end{aligned}
$$

here the coefficients $f_{i}$ are the same as represented in Eqs.(5.7). The expression of $i \frac{\partial}{\partial t_{2}} \mathbf{a}_{\delta}\left(t_{2}\right)$ can directly be written out from Eq.(6.2) by replacing $\gamma$ by $\delta$. When this expression and the one given above are inserted into Eq.(6.1), we are led to

$$
\begin{aligned}
& i \frac{\partial}{\partial t_{2}} G\left(\alpha \beta ; \gamma \delta ; t_{1}-t_{2}\right)=-i \delta\left(t_{1}-t_{2}\right) S(\alpha \beta, \gamma \delta) \\
& +\sum_{\rho \sigma \lambda} \omega_{\rho \sigma} \Delta_{\gamma \delta, \sigma \lambda} G\left(\alpha \beta ; \rho \lambda ; t_{1}-t_{2}\right) \\
& +\sum_{\rho \sigma \lambda} G_{1}\left(\alpha \beta ; \rho \sigma \lambda ; t_{1}-t_{2}\right) g_{1}(\rho \sigma \lambda ; \gamma \delta) \\
& +\sum_{\rho \sigma \tau \lambda} G_{2}\left(\alpha \beta ; \rho \sigma \tau \lambda ; t_{1}-t_{2}\right) g_{2}(\rho \sigma \tau \lambda ; \gamma \delta) \\
& +\sum_{\rho \sigma \lambda} G_{3}\left(\alpha \beta ; \rho \sigma \lambda ; t_{1}-t_{2}\right) g_{3}(\rho \sigma \lambda ; \gamma \delta),
\end{aligned}
$$

where $S(\alpha \beta, \gamma \delta)$ was defined in Eq.(5.4), the coefficients $g_{i}$ are the same as those represented in Eq.(5.10) since the following equality holds

$$
g_{i}(\rho, \ldots, \lambda ; \gamma \delta)=g_{i}(\gamma \delta ; \rho, \ldots, \lambda)
$$

and the Green functions $G_{i}$ are defined as follows

$$
\begin{aligned}
& G_{1}\left(\alpha \beta ; \rho \sigma \lambda ; t_{1}-t_{2}\right) \\
& =\left\langle 0^{+}\left|T\left\{\mathbf{a}_{\alpha}\left(t_{1}\right) \mathbf{a}_{\beta}\left(t_{1}\right): \mathbf{a}_{\rho}\left(t_{2}\right) \mathbf{a}_{\sigma}\left(t_{2}\right): \mathbf{a}_{\lambda}\left(t_{2}\right)\right\}\right| 0^{-}\right\rangle, \\
& G_{2}\left(\alpha \beta ; \rho \sigma \tau \lambda ; t_{1}-t_{2}\right) \\
& =\left\langle 0^{+}\left|T\left\{\mathbf{a}_{\alpha}\left(t_{1}\right) \mathbf{a}_{\beta}\left(t_{1}\right): \mathbf{a}_{\rho}\left(t_{2}\right) \mathbf{a}_{\sigma}\left(t_{2}\right) \mathbf{a}_{\tau}\left(t_{2}\right): \mathbf{a}_{\lambda}\left(t_{2}\right)\right\}\right| 0^{-}\right\rangle, \\
& G_{3}\left(\alpha \beta ; \rho \sigma \lambda ; t_{1}-t_{2}\right) \\
& =\left\langle 0^{+}\left|T\left\{\mathbf{a}_{\alpha}\left(t_{1}\right) \mathbf{a}_{\beta}\left(t_{1}\right): \mathbf{c}_{\rho}^{+}\left(t_{2}\right) \mathbf{c}_{\sigma}\left(t_{2}\right): \mathbf{a}_{\lambda}\left(t_{2}\right)\right\}\right| 0^{-}\right\rangle .
\end{aligned}
$$

By the Fourier transformation denoted in Eq.(5.14) and noticing Eq.(5.17), we obtain

$$
\begin{aligned}
& \left(\omega+\omega_{\gamma}+\omega_{\delta}\right) G(\alpha \beta ; \gamma \delta ; \omega)=S(\alpha \beta, \gamma \delta) \\
& -\sum_{\rho \sigma \lambda} G_{1}(\alpha \beta ; \rho \sigma \lambda ; \omega) g_{1}(\rho \sigma \lambda ; \gamma \delta) \\
& -\sum_{\rho \sigma \tau \lambda} G_{2}(\alpha \beta ; \rho \sigma \tau \lambda ; \omega) g_{2}(\rho \sigma \tau \lambda ; \gamma \delta) \\
& -\sum_{\rho \sigma \lambda} G_{3}(\alpha \beta ; \rho \sigma \lambda ; \omega) g_{3}(\rho \sigma \lambda ; \gamma \delta) .
\end{aligned}
$$

According to the same procedure as formulated in Eqs.(6.1)-(6.6), one can derive the equations of motion obeyed by the Green's functions denoted in Eqs.(5.11)-(5.13). Because the same expressions of the differentials $i \frac{\partial}{\partial t_{2}} \mathbf{a}_{\gamma}\left(t_{2}\right)$ and $i \frac{\partial}{\partial t_{2}} \mathbf{a}_{\delta}\left(t_{2}\right)$ are employed in all the derivations, the equations of motion for those Green's functions may immediately be written down by referencing the equation in Eq.(6.6). The equation for the Green's function in Eq.(5.11) is

$$
\begin{aligned}
& \left(\omega+\omega_{\gamma}+\omega_{\delta}\right) G_{1}(\rho \sigma \lambda ; \gamma \delta ; \omega)=S_{1}(\rho \sigma \lambda, \gamma \delta) \\
& -\sum_{\mu \nu \tau} G_{11}(\rho \sigma \lambda ; \mu \nu \tau ; \omega) g_{1}(\mu \nu \tau ; \gamma \delta) \\
& -\sum_{\mu \nu \tau \kappa} G_{12}(\rho \sigma \lambda ; \mu \nu \tau \kappa ; \omega) g_{2}(\mu \nu \tau \kappa ; \gamma \delta) \\
& -\sum_{\mu \nu \tau}^{\mu} G_{13}(\rho \sigma \lambda ; \mu \nu \tau ; \omega) g_{3}(\mu \nu \tau ; \gamma \delta),
\end{aligned}
$$


where

$$
S_{1}(\rho \sigma \lambda, \gamma \delta)=\left\langle 0^{+}\left|\left[: \mathbf{a}_{\rho} \mathbf{a}_{\sigma}: \mathbf{a}_{\lambda}, \mathbf{a}_{\gamma} \mathbf{a}_{\delta}\right]\right| 0^{-}\right\rangle,
$$

$G_{11}(\rho \sigma \lambda ; \mu \nu \tau ; \omega), G_{12}(\rho \sigma \lambda ; \mu \nu \tau \kappa ; \omega)$ and $G_{13}(\rho \sigma \lambda ; \mu \nu \tau ; \omega)$ are the Fourier transforms of the following Green's functions

$$
\begin{aligned}
& G_{11}\left(\rho \sigma \lambda ; \mu \nu \tau ; t_{1}-t_{2}\right) \\
& =\left\langle 0^{+}\left|T\left\{: \mathbf{a}_{\rho}\left(t_{1}\right) \mathbf{a}_{\sigma}\left(t_{1}\right): \mathbf{a}_{\lambda}\left(t_{1}\right): \mathbf{a}_{\mu}\left(t_{2}\right) \mathbf{a}_{\nu}\left(t_{2}\right): \mathbf{a}_{\tau}\left(t_{2}\right)\right\}\right| 0^{-}\right\rangle \\
& G_{12}\left(\rho \sigma \lambda ; \mu \nu \tau \kappa ; t_{1}-t_{2}\right) \\
& =\left\langle 0^{+}\left|T\left\{: \mathbf{a}_{\rho}\left(t_{1}\right) \mathbf{a}_{\sigma}\left(t_{1}\right): \mathbf{a}_{\lambda}\left(t_{1}\right): \mathbf{a}_{\mu}\left(t_{2}\right) \mathbf{a}_{\nu}\left(t_{2}\right) \mathbf{a}_{\tau}\left(t_{2}\right): \mathbf{a}_{\kappa}\left(t_{2}\right)\right\}\right| 0^{-}\right\rangle \\
& G_{13}\left(\rho \sigma \lambda ; \mu \nu \tau ; t_{1}-t_{2}\right) \\
& =\left\langle 0^{+}\left|T\left\{: \mathbf{a}_{\rho}\left(t_{1}\right) \mathbf{a}_{\sigma}\left(t_{1}\right): \mathbf{a}_{\lambda}\left(t_{1}\right): \mathbf{c}_{\mu}^{+}\left(t_{2}\right) \mathbf{c}_{\nu}\left(t_{2}\right): \mathbf{a}_{\tau}\left(t_{2}\right)\right\}\right| 0^{-}\right\rangle
\end{aligned}
$$

For the the Green's function in Eq.(5.12), we can write

$$
\begin{aligned}
& \left(\omega+\omega_{\gamma}+\omega_{\delta}\right) G_{2}(\rho \sigma \tau \lambda ; \gamma \delta ; \omega)=S_{2}(\rho \sigma \tau \lambda, \gamma \delta) \\
& -\sum_{\mu \nu \theta} G_{21}(\rho \sigma \tau \lambda ; \mu \nu \theta ; \omega) g_{1}(\mu \nu \theta ; \gamma \delta) \\
& -\sum_{\mu \nu \theta \kappa} G_{22}(\rho \sigma \tau \lambda ; \mu \nu \kappa \theta ; \omega) g_{2}(\mu \nu \kappa \theta ; \gamma \delta) \\
& -\sum_{\mu \nu \theta} G_{23}(\rho \sigma \tau \lambda ; \mu \nu \theta ; \omega) g_{3}(\mu \nu \theta ; \gamma \delta),
\end{aligned}
$$

where

$$
S_{2}(\rho \sigma \tau \lambda, \gamma \delta)=\left\langle 0^{+}\left|\left[: \mathbf{a}_{\rho} \mathbf{a}_{\sigma} \mathbf{a}_{\tau}: \mathbf{a}_{\lambda}, \mathbf{a}_{\gamma} \mathbf{a}_{\delta}\right]\right| 0^{-}\right\rangle
$$

and the Green's functions on the RHS of Eq.(6.10) are defined as

$$
\begin{aligned}
& G_{21}\left(\rho \sigma \tau \lambda ; \mu \nu \theta ; t_{1}-t_{2}\right) \\
& =\left\langle 0^{+}\left|T\left\{: \mathbf{a}_{\rho}\left(t_{1}\right) \mathbf{a}_{\sigma}\left(t_{1}\right) \mathbf{a}_{\tau}\left(t_{1}\right): \mathbf{a}_{\lambda}\left(t_{1}\right): \mathbf{a}_{\mu}\left(t_{2}\right) \mathbf{a}_{\nu}\left(t_{2}\right): \mathbf{a}_{\theta}\left(t_{2}\right)\right\}\right| 0^{-}\right\rangle, \\
& G_{22}\left(\rho \sigma \tau \lambda ; \mu \nu \kappa \theta ; t_{1}-t_{2}\right) \\
& =\left\langle 0^{+}\left|T\left\{: \mathbf{a}_{\rho}\left(t_{1}\right) \mathbf{a}_{\sigma}\left(t_{1}\right) \mathbf{a}_{\tau}\left(t_{1}\right): \mathbf{a}_{\lambda}\left(t_{1}\right): \mathbf{a}_{\mu}\left(t_{2}\right) \mathbf{a}_{\nu}\left(t_{2}\right) \mathbf{a}_{\kappa}\left(t_{2}\right): \mathbf{a}_{\theta}\left(t_{2}\right)\right\}\right| 0^{-}\right\rangle, \\
& G_{23}\left(\rho \sigma \tau \lambda ; \mu \nu \theta ; t_{1}-t_{2}\right) \\
& =\left\langle 0^{+}\left|T\left\{: \mathbf{a}_{\rho}\left(t_{1}\right) \mathbf{a}_{\sigma}\left(t_{1}\right) \mathbf{a}_{\tau}\left(t_{1}\right): \mathbf{a}_{\lambda}\left(t_{1}\right): \mathbf{c}_{\mu}^{+}\left(t_{2}\right) \mathbf{c}_{\nu}\left(t_{2}\right): \mathbf{a}_{\theta}\left(t_{2}\right)\right\}\right| 0^{-}\right\rangle .
\end{aligned}
$$

For the Green's function in Eq.(5.13), we have the equation

$$
\begin{aligned}
& \left(\omega+\omega_{\gamma}+\omega_{\delta}\right) G_{3}(\rho \sigma \lambda ; \gamma \delta ; \omega)=S_{3}(\rho \sigma \lambda, \gamma \delta) \\
& -\sum_{\mu \nu \tau} G_{31}(\rho \sigma \lambda ; \mu \nu \tau ; \omega) g_{1}(\mu \nu \tau ; \gamma \delta) \\
& -\sum_{\mu \nu \tau \kappa} G_{32}(\rho \sigma \lambda ; \mu \nu \tau \kappa ; \omega) g_{2}(\mu \nu \tau \kappa ; \gamma \delta) \\
& -\sum_{\mu \nu \tau} G_{33}(\rho \sigma \lambda ; \mu \nu \tau ; \omega) g_{3}(\mu \nu \tau ; \gamma \delta)
\end{aligned}
$$

where

$$
S_{3}(\rho \sigma \lambda, \gamma \delta)=\left\langle 0^{+}\left|\left[: \mathbf{c}_{\rho}^{+} \mathbf{c}_{\sigma}: \mathbf{a}_{\lambda}, \mathbf{a}_{\gamma} \mathbf{a}_{\delta}\right]\right| 0^{-}\right\rangle
$$

and the Green's functions on the RHS of Eq.(6.13) are defined by

$$
\begin{aligned}
& G_{31}\left(\rho \sigma \lambda ; \mu \nu \tau ; t_{1}-t_{2}\right) \\
& =\left\langle 0^{+}\left|T\left\{: \mathbf{c}_{\rho}^{+}\left(t_{1}\right) \mathbf{c}_{\sigma}\left(t_{1}\right): a_{\lambda}\left(t_{1}\right): a_{\mu}\left(t_{2}\right) a_{\nu}\left(t_{2}\right): a_{\tau}\left(t_{2}\right)\right\}\right| 0^{-}\right\rangle, \\
& G_{32}\left(\rho \sigma \lambda ; \mu \nu \tau \kappa ; t_{1}-t_{2}\right) \\
& =\left\langle 0^{+}\left|T\left\{: \mathbf{c}_{\rho}^{+}\left(t_{1}\right) \mathbf{c}_{\sigma}\left(t_{1}\right): \mathbf{a}_{\lambda}\left(t_{1}\right): \mathbf{a}_{\mu}\left(t_{2}\right) \mathbf{a}_{\nu}\left(t_{2}\right) \mathbf{a}_{\tau}\left(t_{2}\right): \mathbf{a}_{\kappa}\left(t_{2}\right)\right\}\right| 0^{-}\right\rangle, \\
& G_{33}\left(\rho \sigma \lambda ; \mu \nu \tau ; t_{1}-t_{2}\right) \\
& =\left\langle 0^{+}\left|T\left\{: \mathbf{c}_{\rho}^{+}\left(t_{1}\right) \mathbf{c}_{\sigma}\left(t_{1}\right): \mathbf{a}_{\lambda}\left(t_{1}\right): \mathbf{c}_{\mu}^{+}\left(t_{2}\right) \mathbf{c}_{\nu}\left(t_{2}\right): \mathbf{a}_{\tau}\left(t_{2}\right)\right\}\right| 0^{-}\right\rangle .
\end{aligned}
$$

Now we are ready to derive the interaction kernels. Multiplying the both sides of Eq.(5.19) with $\left(\omega+\omega_{\gamma}+\omega_{\delta}\right)$ and then applying Eqs.(6.6) and (6.7), we have 


$$
\begin{aligned}
& \sum_{\rho \sigma} \Lambda_{1}(\alpha \beta ; \rho \sigma ; \omega)\left[S(\rho \sigma, \gamma \delta)-\sum_{\mu \nu \lambda} G_{1}(\rho \sigma ; \mu \nu \lambda ; \omega) g_{1}(\mu \nu \lambda ; \gamma \delta)\right. \\
& -\sum_{\mu \nu \tau \lambda} G_{2}(\rho \sigma ; \mu \nu \tau \lambda ; \omega) g_{2}(\mu \nu \tau \lambda ; \gamma \delta) \\
& \left.-\sum_{\mu \nu \lambda} G_{3}(\rho \sigma ; \mu \nu \lambda ; \omega) g_{3}(\mu \nu \lambda ; \gamma \delta)\right] \\
= & \sum_{\rho \sigma \lambda} g_{1}(\alpha \beta ; \rho \sigma \lambda)\left[S_{1}(\rho \sigma \lambda, \gamma \delta)-\sum_{\mu \nu \tau} G_{11}(\rho \sigma \lambda ; \mu \nu \tau ; \omega) g_{1}(\mu \nu \tau ; \gamma \delta)\right. \\
& -\sum_{\mu \nu \tau \kappa} G_{12}(\rho \sigma \lambda ; \mu \nu \tau \kappa ; \omega) g_{2}(\mu \nu \tau \kappa ; \gamma \delta) \\
& \left.-\sum_{\mu \nu \tau} G_{13}(\rho \sigma \lambda ; \mu \nu \tau ; \omega) g_{3}(\mu \nu \tau ; \gamma \delta)\right] .
\end{aligned}
$$

In order to obtain the expression of the kernel $\Lambda_{1}(\alpha \beta ; \rho \sigma ; \omega)$ from the above equation, it is necessary to eliminate the kernel in the second, third and fourth terms on the left hand side (LHS) of the above equation. Considering that the Green's function $G(\alpha \beta ; \gamma \delta ; \omega)$, as a matrix $G$, has an inverse $G^{-1}(\alpha \beta ; \gamma \delta ; \omega)$, then from Eq.(5.19) we can get

$$
\Lambda_{1}(\alpha \beta ; \gamma \delta ; \omega)=\sum_{\rho \sigma \lambda} \sum_{\mu \nu} g_{1}(\alpha \beta ; \rho \sigma \lambda) G_{1}(\rho \sigma \lambda ; \mu \nu ; \omega) G^{-1}(\mu \nu ; \gamma \delta ; \omega) .
$$

Substituting Eq.(6.17) into the second, third and fourth terms on the LHS of Eq.(6.16), then moving these terms to the RHS of Eq.(6.16) and finally acting on the both sides of that equation with the inverse of the matrix $S(\rho \sigma, \gamma \delta)$ which is assumed to exist, we eventually arrive at

$$
\begin{aligned}
\Lambda_{1}(\alpha \beta ; \gamma \delta ; \omega)= & \Lambda_{1}^{(1)}(\alpha \beta ; \gamma \delta ; \omega)+\Lambda_{1}^{(2)}(\alpha \beta ; \gamma \delta ; \omega) \\
& +\Lambda_{1}^{(3)}(\alpha \beta ; \gamma \delta ; \omega),
\end{aligned}
$$

where

$$
\begin{aligned}
& \Lambda_{1}^{(1)}(\alpha \beta ; \gamma \delta ; \omega)=\sum_{\rho \sigma \lambda} \sum_{\mu \nu} g_{1}(\alpha \beta ; \rho \sigma \lambda) S_{1}(\rho \sigma \lambda, \mu \nu) S^{-1}(\mu \nu, \gamma \delta), \\
& \Lambda_{1}^{(2)}(\alpha \beta ; \gamma \delta ; \omega) \\
&=-\sum_{\rho \sigma \lambda} \sum_{\theta \pi} g_{1}(\alpha \beta ; \rho \sigma \lambda)\left\{\sum_{\mu \nu \tau} G_{11}(\rho \sigma \lambda ; \mu \nu \tau ; \omega) g_{1}(\mu \nu \tau ; \theta \pi)\right. \\
&+\sum_{\mu \nu \tau \kappa} G_{12}(\rho \sigma \lambda ; \mu \nu \tau \kappa ; \omega) g_{2}(\mu \nu \tau \kappa ; \theta \pi) \\
&\left.+\sum_{\mu \nu \tau} G_{13}(\rho \sigma \lambda ; \mu \nu \tau ; \omega) g_{3}(\mu \nu \tau ; \theta \pi)\right\} S^{-1}(\theta \pi, \gamma \delta),
\end{aligned}
$$

and

$$
\begin{aligned}
& \Lambda_{1}^{(3)}(\alpha \beta ; \gamma \delta ; \omega) \\
= & \sum_{\rho \sigma \lambda} \sum_{\mu \nu} \sum_{\epsilon \iota} \sum_{\theta \pi} g_{1}(\alpha \beta ; \rho \sigma \lambda) G_{1}(\rho \sigma \lambda ; \mu \nu ; \omega) G^{-1}(\mu \nu ; \epsilon \iota ; \omega) \\
& \times\left\{\sum_{\xi \eta \zeta} G_{1}(\epsilon \iota ; \xi \eta \zeta ; \omega) g_{1}(\xi \eta \zeta ; \theta \pi)\right. \\
& +\sum_{\xi \eta \zeta \chi} G_{2}(\epsilon \iota ; \xi \eta \zeta \chi ; \omega) g_{2}(\xi \eta \zeta \chi ; \theta \pi) \\
& \left.+\sum_{\xi \eta \zeta} G_{3}(\epsilon \iota ; \xi \eta \zeta ; \omega) g_{3}(\xi \eta \zeta ; \theta \pi)\right\} S^{-1}(\theta \pi, \gamma \delta) .
\end{aligned}
$$


By the same method as described above, the kernel $\Lambda_{2}(\alpha \beta ; \gamma \delta ; \omega)$ can be derived from the relation in Eq.(5.20) by using the equations in Eq.(6.6) and (6.10). The result is as follows

$$
\Lambda_{2}(\alpha \beta ; \gamma \delta ; \omega)=\Lambda_{2}^{(1)}(\alpha \beta ; \gamma \delta ; \omega)+\Lambda_{2}^{(2)}(\alpha \beta ; \gamma \delta ; \omega)+\Lambda_{2}^{(3)}(\alpha \beta ; \gamma \delta ; \omega)
$$

where

$$
\begin{aligned}
\Lambda_{2}^{(1)}(\alpha \beta ; \gamma \delta ; \omega)=\sum_{\rho \sigma \tau \lambda} \sum_{\mu \nu} g_{2}(\alpha \beta ; \rho \sigma \tau \lambda) S_{2}(\rho \sigma \tau \lambda ; \mu \nu ; \omega) S^{-1}(\mu \nu, \gamma \delta), \\
\quad \Lambda_{2}^{(2)}(\alpha \beta ; \gamma \delta ; \omega) \\
=-\sum_{\rho \sigma \tau \lambda} \sum_{\theta \pi} g_{2}(\alpha \beta ; \rho \sigma \tau \lambda)\left\{\sum_{\mu \nu \varepsilon} G_{21}(\rho \sigma \tau \lambda ; \mu \nu \varepsilon ; \omega) g_{1}(\mu \nu \varepsilon ; \theta \pi)\right. \\
\quad+\sum_{\mu \nu \varepsilon \kappa} G_{22}(\rho \sigma \tau \lambda ; \mu \nu \varepsilon \varkappa ; \omega) g_{2}(\mu \nu \varepsilon \varkappa ; \theta \pi) \\
\left.\quad-\sum_{\mu \nu \varepsilon} G_{23}(\rho \sigma \tau \lambda ; \mu \nu \varepsilon ; \omega) g_{3}(\mu \nu \varepsilon ; \theta \pi)\right\} S^{-1}(\theta \pi, \gamma \delta),
\end{aligned}
$$

and

$$
\begin{aligned}
& \Lambda_{2}^{(3)}(\alpha \beta ; \gamma \delta ; \omega) \\
= & \sum_{\rho \sigma \tau \lambda} \sum_{\mu \nu} \sum_{\epsilon \iota} \sum_{\theta \pi} g_{2}(\alpha \beta ; \rho \sigma \tau \lambda) G_{2}(\rho \sigma \tau \lambda ; \mu \nu ; \omega) G^{-1}(\mu \nu ; \epsilon \iota ; \omega) \\
& \times\left\{\sum_{\xi \eta \zeta} G_{1}(\epsilon \iota ; \xi \eta \zeta ; \omega) g_{1}(\xi \eta \zeta ; \theta \pi)\right. \\
& +\sum_{\xi \eta \zeta \kappa} G_{2}(\epsilon \iota ; \xi \eta \zeta \chi ; \omega) g_{2}(\xi \eta \zeta \chi ; \theta \pi) \\
& \left.+\sum_{\xi \eta \zeta} G_{3}(\epsilon \iota ; \xi \eta \zeta ; \omega) g_{3}(\xi \eta \zeta ; \theta \pi)\right\} S^{-1}(\theta \pi, \gamma \delta) .
\end{aligned}
$$

Analogously, the kernel $\Lambda_{3}(\alpha \beta ; \gamma \delta ; \omega)$ may be derived from Eqs.(5.21), (6.6) and (6.13). The result is shown below

$$
\begin{aligned}
& \Lambda_{3}(\alpha \beta ; \gamma \delta ; \omega) \\
= & \Lambda_{3}^{(1)}(\alpha \beta ; \gamma \delta ; \omega)+\Lambda_{3}^{(2)}(\alpha \beta ; \gamma \delta ; \omega)+\Lambda_{3}^{(3)}(\alpha \beta ; \gamma \delta ; \omega),
\end{aligned}
$$

where

$$
\begin{aligned}
& \Lambda_{3}^{(1)}(\alpha \beta ; \gamma \delta ; \omega)=\sum_{\rho \sigma \lambda} \sum_{\mu \nu} g_{3}(\alpha \beta ; \rho \sigma \lambda) S_{3}(\rho \sigma \lambda, \mu \nu) S^{-1}(\mu \nu, \gamma \delta), \\
&=-\sum_{\rho \sigma \tau \lambda}^{(2)} \sum_{\theta \pi} g_{3}(\alpha \beta ; \rho \sigma \lambda)\left\{\sum_{\mu \nu \tau} G_{31}(\rho \sigma \lambda ; \mu \nu \tau ; \omega) g_{1}(\mu \nu \tau ; \theta \pi)\right. \\
& \quad+\sum_{\mu \nu \tau \kappa} G_{32}(\rho \sigma \lambda ; \mu \nu \tau \kappa ; \omega) g_{2}(\mu \nu \tau \kappa ; \theta \pi) \\
&\left.\quad+\sum_{\mu \nu \tau} G_{33}(\rho \sigma \lambda ; \mu \nu \tau ; \omega) g_{3}(\mu \nu \tau ; \theta \pi)\right\} S^{-1}(\theta \pi, \gamma \delta), \\
& \Lambda_{3}^{(3)}(\alpha \beta ; \gamma \delta ; \omega) \\
&= \sum_{\rho \sigma \lambda} \sum_{\mu \nu} \sum_{\epsilon \iota} \sum_{\theta \pi} g_{3}(\alpha \beta ; \rho \sigma \lambda) G_{3}(\rho \sigma \lambda ; \mu \nu ; \omega) G^{-1}(\mu \nu ; \epsilon \iota ; \omega)
\end{aligned}
$$




$$
\begin{aligned}
& \times\left\{\sum_{\xi \eta \zeta} G_{1}(\epsilon \iota ; \xi \eta \zeta ; \omega) g_{1}(\xi \eta \zeta ; \theta \pi)\right. \\
& +\sum_{\xi \eta \zeta \kappa} G_{2}(\epsilon \iota ; \xi \eta \zeta \chi ; \omega) g_{2}(\xi \eta \zeta \chi ; \theta \pi) \\
& \left.+\sum_{\xi \eta \zeta} G_{3}(\epsilon \iota ; \xi \eta \zeta ; \omega) g_{3}(\xi \eta \zeta ; \theta \pi)\right\} S^{-1}(\theta \pi, \gamma \delta) .
\end{aligned}
$$

Based on the expressions described in Eqs.(6.18)-(6.29), the total kernel can easily be written out. Using the matrix notation for the Green's functions and the other functions, the kernel is represented as

$$
\begin{aligned}
K & =\sum_{i, j=1}^{3} \Lambda_{i}^{(i)} \\
& =\left\{\sum_{i=1}^{3} g_{i} S_{i}-\sum_{i, j=1}^{3} g_{i} G_{i j} g_{j}+\sum_{i, j=1}^{3} g_{i} G_{i} G^{-1} G_{j} g_{j}\right\} S^{-1} .
\end{aligned}
$$

This is just the closed expression of the interaction kernel. According to the general argument as presented in Refs. [33,34,39,40], the third term in the above expression plays the role of eliminating all the B-S reducible (twoparticle reducible) diagrams contained in the first two terms. In fact, the relations in Eqs.(5.19)-(5.21) and the following ones

$$
G_{j} g_{j}=G \Lambda_{j}
$$

are inserted into the last term in Eq.(6.30), it is seen that

$$
\sum_{i, j=1}^{3} g_{i} G_{i} G^{-1} G_{j} g_{j}=\sum_{i, j=1}^{3} \Lambda_{i} G \Lambda_{j}=K G K,
$$

which exhibits the typical structure of the B-S reducible part of the interaction kernel. Therefore, the kernel shown in Eq.(6.30) is truly B-S irreducible, consistent with the conventional concept. The equation in Eq.(5.28) and the kernel in Eq.(6.30) will be employed, in the next paper, to calculate the glueball spectrum in the ladder approximation.

\section{CONCLUDING REMARKS}

In this paper, the exact three-dimensional relativistic equation for two gluon glueball states and its interaction kernel have been derived from the QCD with massive gluons. When the gluon mass tends to zero, the equation and its kernel will naturally go over to the ones for the QCD with massless gluons. As shown in Eq.(5.28), The equation derived is a standard eigenvalue equation of Schrödinger-type. In the position space it appears to be a set of first-order differential equations. This kind of equation was given for fermion systems [39,40], but never formulated for boson systems in the past. It should be noted that since the equation is derived in a special equal-time Lorentz frame, it is certainly not Lorentz-covariant even though the equation is rigorous and includes all the retardation effect in it. In order to derive a Lorentz-covariant equation, one may start from the four-time Green's function

$$
G_{\alpha \beta \gamma \delta}\left(T, t ; T^{\prime}, t^{\prime}\right)=\left\langle 0^{+}\left|T\left\{\mathbf{a}_{\alpha}\left(t_{1}\right) \mathbf{a}_{\beta}\left(t_{2}\right) \mathbf{a}_{\gamma}\left(t_{3}\right) \mathbf{a}_{\delta}\left(t_{4}\right)\right\}\right| 0^{-}\right\rangle,
$$

where

$$
\begin{aligned}
T & =\frac{1}{2}\left(t_{1}+t_{2}\right), t=t_{1}-t_{2}, \\
T^{\prime} & =\frac{1}{2}\left(t_{3}+t_{4}\right), t^{\prime}=t_{3}-t_{4} .
\end{aligned}
$$

Differentiating the above Green's function with respect to $t_{1}$ and $t_{2}$ and employing the expression of the differential $i \frac{\partial}{\partial t_{1}} \mathbf{a}_{\alpha}\left(t_{1}\right)$ as shown in Eq. (5.6) and the similar expression for $i \frac{\partial}{\partial t_{2}} \mathbf{a}_{\beta}\left(t_{2}\right)$, one may obtain two equations for the Green's function $G_{\alpha \beta \gamma \delta}\left(T, t ; T^{\prime}, t^{\prime}\right)$. Adding the both equations and subtracting one equation from another, following the same procedure as formulated in Sec.V, it is easy to derive two equations satisfied by the B-S amplitude $\chi_{\alpha \beta}(T, t)$ 
which respectively describe the evolutions of the state with respect to the center of mass time $T$ and the relative time $t$. In the equal-time frame, owing to the relative time being absent, we are left only with the equation with respect to the center of mass time as given in Sec.V. It is interesting to note that either the equation with respect to $T$ or the equation with respect to $t$, appears to be a first order differential equation of Schrödinger-type in the position space whose solutions are determined merely by the initial condition of the B-S amplitudes at the time origin. This is an essential feature of the equations mentioned above which is different from the B-S equation. The latter equation is a higher order differential equation and hence, like the Klein-Gordon equation, has unphysical solutions with negative norm as pointed out in the previous literature [35]. This is because the solutions of B-S equation are determined not only by the initial amplitudes at the time origin, but also by the time-differentials of the amplitudes at the time origin.

Another point we would like to note is that unlike the Dyson-Schwinger equation, the relativistic equation derived in this paper is of a closed form. In particular, the interaction kernel in the equation is given a closed expression. The expression contains only a few types of Green's functions and vacuum expectation values of the operator commutators. They are unambiguously defined in the Heisenberg picture and each of them can independently be calculated by the perturbation method without concerning other Green's functions. Especially, the kernel represents all the interactions taking place in the bound states and, therefore, are suitable for nonperturbative investigations because the Green's functions and vacuum expectation values of the commutators, in principle, are able to be evaluated by a certain nonperturbative method as suggested by the lattice gauge approach.

At last, it would be pointed out that although the equation in Sec.V and the kernel in Sec.VI are derived in the angular momentum representation, they suit to formulate the equation and the kernel in the momentum representation as long as the angular momentum quantum numbers in the indices $\alpha, \beta, \cdots$ are replaced by the momentum ones. That is to say, the equation and the kernel formally remain unchanged in the both representations.

\section{ACKNOWLEDGEMENT}

The authors are grateful to professor Shi-Shu Wu for useful discussions. This work was supported in part by National Natural Science Foundation of China.

\section{APPENDIX: SPHERICAL SPINORS IN THE ANGULAR MOMENTUM REPRESENTATION}

In this appendix, we intend to give a derivation of the spherical Dirac spinors which are used, as basis functions, to establish the angular momentum representation for fermion fields. It is well-known that in the relativistic case, unlike the helicity, the spin of a free fermion is not a good quantum number. However, the total angular momentum operator of the fermion commutes with the Hamiltonian. Therefore, it is meaningful to discuss eigenfunctions of the total angular momentum which satisfy Dirac equation. Let us start from the positive energy spinor $u_{s}(\vec{p})$ which is the solution to the Dirac equation $\left(i \partial^{\mu} p_{\mu}-m\right) u(\vec{p})=0$. This spinor is taken to be [46]

$$
u_{s}(\vec{p})=\sqrt{\frac{\varepsilon+m}{2 \varepsilon}}\left(\frac{1}{\frac{\vec{\sigma} \cdot \vec{p}}{\varepsilon+m}}\right) \varphi_{s}
$$

which is normalized in such a fashion: $u_{s}^{+}(\vec{p}) u_{s}(\vec{p})=1$. The negative energy spinor can be given by the charge conjugation $v_{s}(\vec{p})=C \bar{u}_{s}(\vec{p})^{T}$. Suppose $Y_{l m}(\widehat{p})$ with $\widehat{p}=\vec{p} /|\vec{p}|=(\theta, \varphi)$ and $\varphi_{s}$ are the orbital angular momentum and the spin eigenfunctions respectively; the total angular momentum eigenfunctions may be constructed in the momentum space by the $\mathrm{C}-\mathrm{G}$ coupling

$$
\Omega_{J M}^{l}(\widehat{p})=\sum_{l s} C_{l m \frac{1}{2} s}^{J M} Y_{l m}(\widehat{p}) \varphi_{s}
$$

where $s=\frac{\sigma}{2}, \sigma= \pm 1, l=J \pm \frac{\sigma}{2}$.Noticing the representation

$$
\varphi_{\frac{1}{2}}=\left(\begin{array}{l}
0 \\
1
\end{array}\right), \varphi_{-\frac{1}{2}}=\left(\begin{array}{l}
1 \\
0
\end{array}\right)
$$

and employing the explicit expressions of the C-G coupling coefficients for different values of $\sigma$ which may be found in the textbook, one may derive from (A.2) the expression as follows 


$$
\Omega_{J M}^{\sigma}(\widehat{p})=\left(\begin{array}{c}
\sigma \sqrt{\frac{J+\sigma\left(M-\frac{1}{2}\right)+\frac{1}{2}}{2 J-l+1}} Y_{J-\frac{\sigma}{2}, M-\frac{1}{2}}(\widehat{p}) \\
\sqrt{\frac{J-\sigma\left(M+\frac{1}{2}\right)+\frac{1}{2}}{2 J-\sigma+1}} Y_{J-\frac{\sigma}{2}, M+\frac{1}{2}}(\widehat{p})
\end{array}\right) .
$$

By making use of the Dirac spinor in (A.1) and the eigenfunctions represented in (A.2) or in (A.4) with respect to the momentum $\vec{p}$, we may construct the spherical Dirac spinor in the position space through the following Fourier transformation

$$
u_{J M}^{\sigma}(p \vec{x})=\int d \widehat{p} \frac{e^{i \vec{p} \cdot \vec{x}}}{(2 \pi)^{3 / 2}} p u(\vec{p}) \Omega_{J M}^{\sigma}(\widehat{p}) .
$$

Substituting the expansion

$$
e^{i \vec{p} \cdot \vec{x}}=4 \pi \sum_{l m} i^{l} j_{l}(p r) Y_{l m}^{*}(\widehat{p}) Y_{l m}(\widehat{x})
$$

and the expressions written in (A.1) and (A.4) into (A.5), considering

$$
\vec{\sigma} \cdot \widehat{p}=\left(\begin{array}{ll}
\cos \theta & \sin \theta e^{-i \varphi} \\
\sin \theta e^{i \varphi} & -\cos \theta
\end{array}\right)
$$

and

$$
\vec{\sigma} \cdot \widehat{p} \Omega_{J M}^{\sigma}(\widehat{p})=-\Omega_{J M}^{-\sigma}(\widehat{p}),
$$

which is easily proved by utilizing the familiar recursion formulas for the spherical harmornic functions, it is not difficult to derive the expression shown in Eq.(3.22). The expression of the function $v_{J M}^{\sigma}(p \vec{x})$ may be derived by the charge conjugation denoted in Eq.(3.34). The result was written in Eq.(3.23). It would be noted that the

eigenfunction $\Omega_{J M}^{\sigma}(\widehat{x})$ in Eq.(3.24) which is defined in the position space and appears in Eqs.(3.22) and (3.23) is of the same form as the function $\Omega_{J M}^{\sigma}(\widehat{p})$ in (A.4) which is defined in the momentum space.

\section{REFERENCE}

[1] H.Fritzsch and M. Gell-Mann, in Proc.16 intern. Conf. on High Energy Physics, FNAL, Batavia, IL, P.135 (1972).

[2] H. Fritzsch and P.Minkowski, Nuovo Cimento 30A, 393 (1975).

[3] Crystal Barrel Collaboration, Phys. Lett. B323, 223 (1994).

[4] V. V. Anisovich, et. al., Phys. Rev. D50, 1972 (1994).

[5] S. Bhatnagar and A. N. Mitra, Nuovo Cim. A104, 925 (1991).

[6] R. M. Baltrusaitis, et. al., Phys. Rev. Lett. 56, 107 (1986).

[7] J. Z. Bai, et. al., Phys. Rev. Lett. 76, 3502 (1996).

[8] R. M. Barnett, et. al., Phys. Rev. D54, 1 (1996).

[9] C. Amsler, et. al., Phys. Lett. B355, 425 (1995); Phys. Lett. B353, 385 (1995).

[10] D. Weingarten, Nucl. Phys. Proc. Suppl. B53, 232 (1997).

[11] D.V. Bugg, M. Peardon, B.S. Zou, Phys. Lett. B486, 49 (2000).

[12] J. Weinstein and N. Isgur, Phys. Rev. D27, 588 (1983); D41, 2236 (1990); D43, 95 (1991).

[13] L. Burakovsky, P.R. Page, Eur. Phys. J. C12, 489 (2000).

[14] J. Sexton, A. Vaccarino and D. Weigarten, Phys. Rev. Lett. 75, 4563 (1995).

[15] V. V. Anisovich, Phys. Lett. B364, 195 (1995).

[16] T. Barnes, Z. Phys. C10, 275 (1981).

[17] J. M. Cornwall and A. Soni, Phys. Lett. B120, 431 (1983).

[18] W. S. Hou and G. G. Wong, Phys. Rev. D67, 034003 (2003).

[19] R. L. Jaffe and K. Johnson, Phys. Lett. B60, 201 (1976); Phys. Rev. Lett. 34, 1645 1976).

[20] T. Barnes, F. E. Close and S. Monagham, Nucl. Phys. B198, 380 (1982).

[21] S. Narision, Z. Phys. C26, 209 (1984); S. Narision, Nucl. Phys. B509, 312 1998).

[22] S. Bhatnagar and A. N. Mitra, Nuovo Cim. A104, 925 (1991).

[23] M. H. Thoma, M. Lüst and H. J. Mang, J. Phys. G: Nucl. Part. Phys. 18, 1125 (1992).

[24] J. Y. Cui, J. M. Wu, H. Y. Jin, Phys. Lett. B424, 381 (1998). 
[25] K.G. Wilson, Phys. Rev. D10, 2445 (1974).

[26] C. Bernard, Phys. Lett. 108B, 431 (1982); Nucl. Phys. B219, 341 (1983).

[27] C. Michael and M. Teper, Nucl. Phys. Lett. B314, 347 (1989).

[28] C. orningstar and M. Peardon, Phys. Rev. D60, 034509 (1999).

[29] C. Liu, Chin. Phys. Lett. 18, 187 (2001).

[30] D. Q. Liu, J. M. Wu, Y. Chen, High Ener. Phys. Nucl. Phys. 26, 222 (2002).

[31] G. B. West, talk given at the Montpelier Conference and Pairs Workshop on QCD, hep-ph/9608258.

[32] W. Ochs, talk at EPS-HEP '99 Conference, July 15-21, 1999, Tampere, Finland, hep-ph/9909241.

[33] E. E. Salpeter and H. A. Bethe, Phys. Rev. 84, 1232 (1951).

[34] J. C. Su, Commun. Theor. Phys. 38, 433 (2002).

[35] N. Nakanishi, Prog. Theor. Phys. Suppl. 42, 1 (1969); Prog. Theor. Phys. Suppl. 95, 1 (1988).

[36] E. E. Salpeter, Phys. Rev. 87,328 (1952).

[37] R. Blankenbecler and R. Sugar, Phys. Rev. 142, 1051 (1969).

[38] I. T. Todorov, Phys. Rev. D3, 2351 (1971).

[39] S. S. Wu, J. Phys. G: Nucl. Part. Phys., 16, 1447 (1990).

[40] J. C. Su and D. Z. Mu, Commun. Theor. Phys. 15, 437 (1991).

[41] M. E. Rose, Multipole Fields, John Wiley \& Sons, New York, 1955.

[42] A. R. Edmonds, Angular Momentumin in Quantum Mechanics, Princeton University Press, 1960.

[43] J. C. Su, Nuovo Cim. B 117, 203 (2002).

[44] L. D. Faddeev and V. N. Popov, Phys. Lett. B25, 29 (1967).

[45] J. C. Su, hep-th/9805193, hep-th/9805194.

[46] C.Itzykson and J-B. Zuber, Quantum Field Theory, McGraw-Hill, New York, 1980. 